\title{
Heavy Vehicle Driver Workload Assessment Task 3: Task Analysis Data Collection
}


This research was supported (in part) by the National Highway Traffic Safety Administration (NHTSA), U.S. D epartment of Transportation, Under Contract Ne. DTNH22-91-C.07003,

The opinions, findings and recommendations contained herein are those of the authors, and do not necessarily represent those of the NHTSA. 


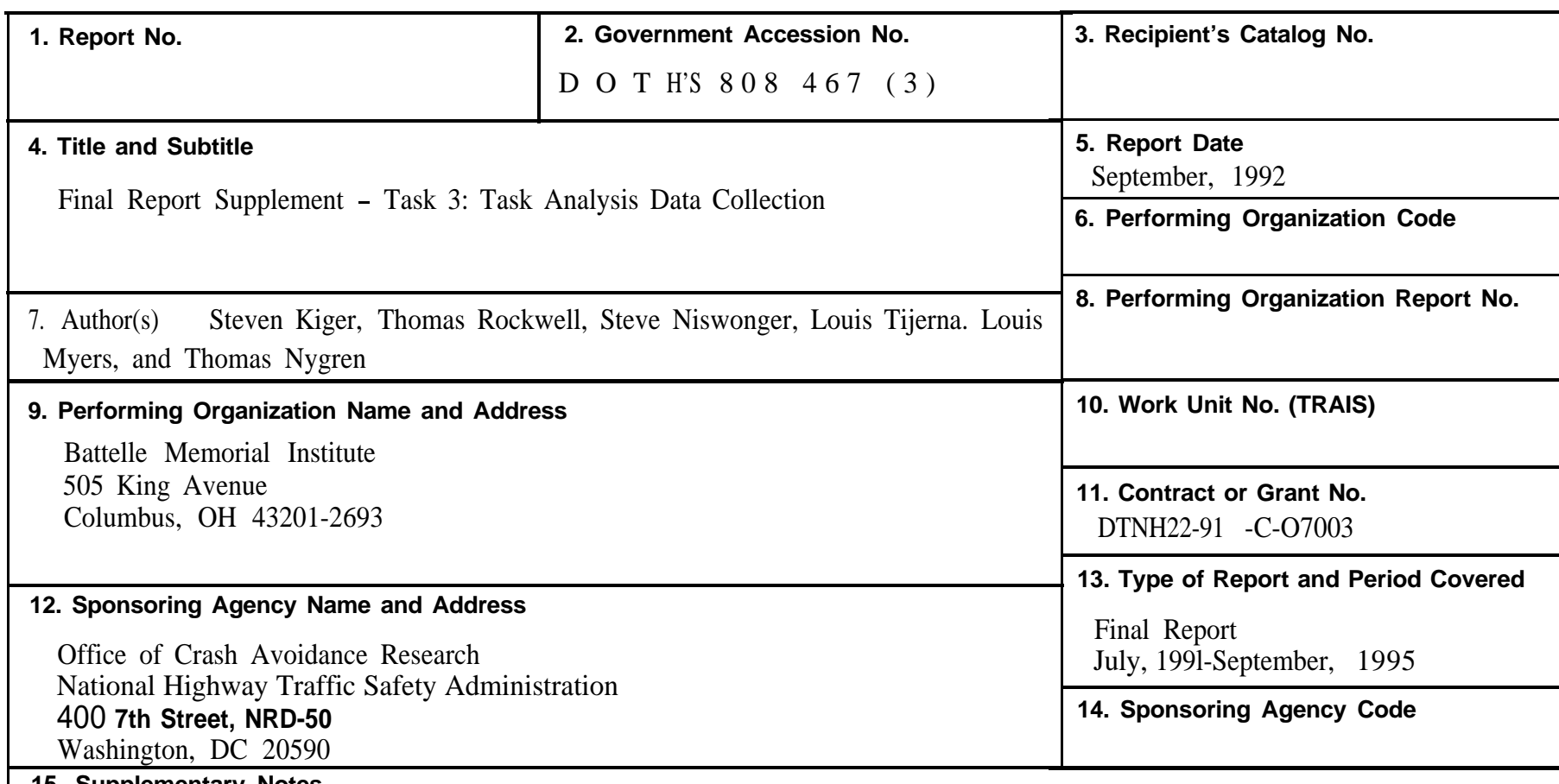

15. Supplementary Notes

Contracting Officer's Technical Representative: Dr. Michael J. Goodman (NRD-50)

\section{Abstract}

This technical report consists of a collection of task analytic data to support heavy vehicle driver workload assessment and protocol development. Data were collected from professional drivers to provide insights into the following issues: the meaning of the term 'workload' to heavy vehicle drivers $(\mathrm{N}=41$ drivers interviewed); the demand placed on drivers $(\mathrm{N}=55)$ by various drving conditions using a psychological scaling approach; the safety criticality and difficulty of selected standard driving tasks ( $\mathrm{N}=30$ drivers assessed); the perceptual, motor, and cognitive loads imposed by various tasks while on the road under various conditions ( $\mathrm{N}=9$ drivers observed over-the-road during revenue runs); and a preliminary analysis of the key features of generic high-technology in-cab devices. When professional truck drivers speak of workload, they tend to talk in terms of time stress or stress caused by delays to their schedules. Various driving conditions were unidimensionally scaled in terms of order of impact on drivers, and those results are presented and discussed. Safety criticality and difficulty assessment results provided some validation for the scaling outcomes. Visual allocation measures in this study suggested that the use of common -in-cab tasks would serve as baseline measures, based on the revenue runs. Finally, key features considered in evaluating voice communication systems, vehicle navigation and route guidance systems, single/integrated displays, and text communicaton devices were determined.

\begin{tabular}{|l|l|l|l|}
\hline 17. Key Words & \multicolumn{3}{l|}{ 18. Distribution Statement } \\
human factors, heavy vehicle, workload, driving conditions, safety, \\
driving tasks, perceptual load, cognitive load, motor load, in-cab \\
devices, navigation, route guidance, display, text
\end{tabular}




\section{TABLE OF CONTENTS}

Page

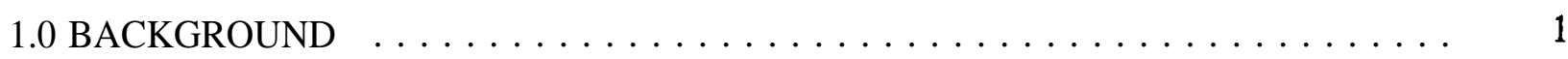

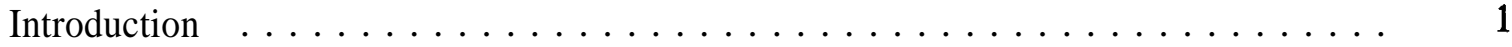

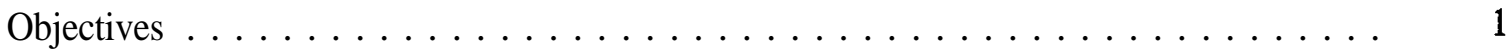

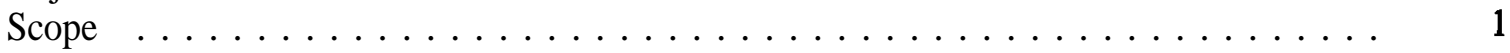

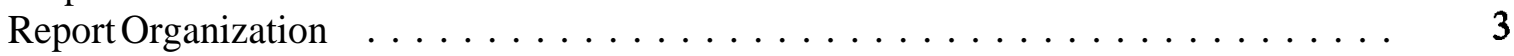

2.0 ASCERTAINING THE DIMENSIONS OF TRUCK DRIVER WORKLOAD . . . . . . . 4

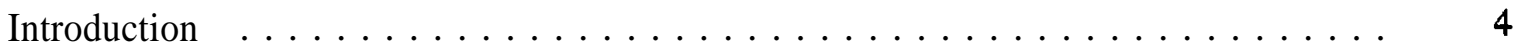

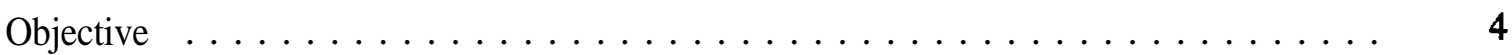

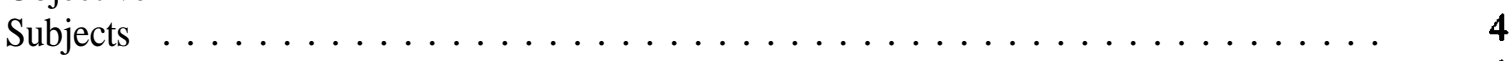

Procedure............................. 4

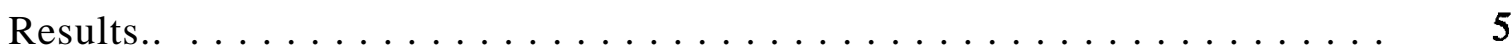

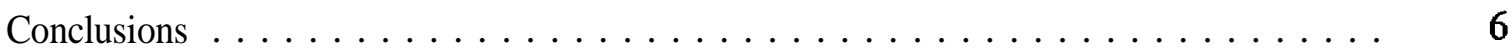

Appendix 2A Truck Driver Interview Guide and Tabulation of Responses Truck Driver Interview Summaries .................... 7

3.0 ASSESSMENT OF DRIVING CONDITION DEMAND $\ldots \ldots \ldots \ldots \ldots \ldots \ldots$

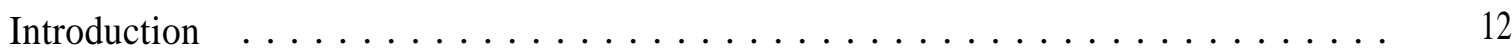

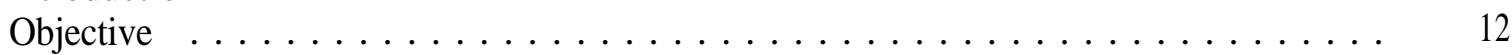

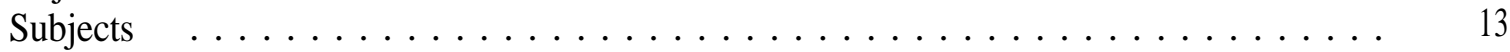

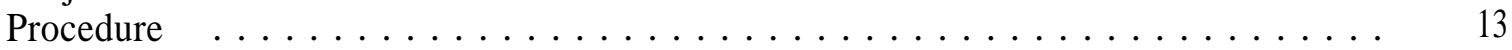

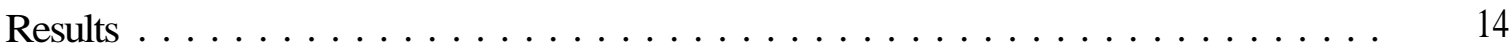

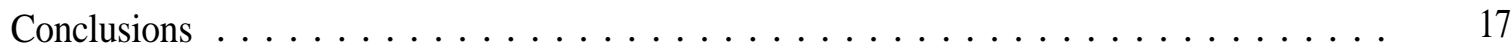

References ............................... 20

Appendix 3A Further Description of Conjoint Analysis . . . . . . . . . . . . . . 21

4.0 DIFFICULTY AND IMPORTANCE RATINGS OF TRUCK DRIVING TASKS

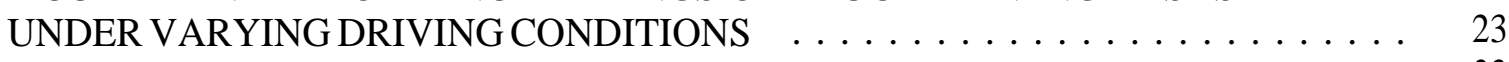

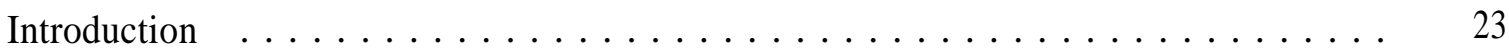

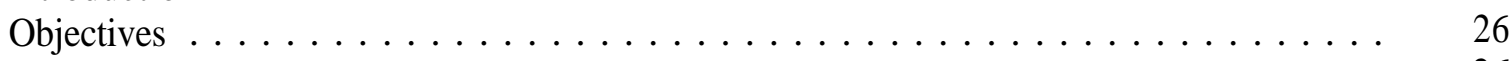

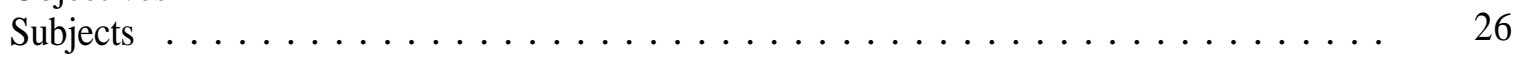

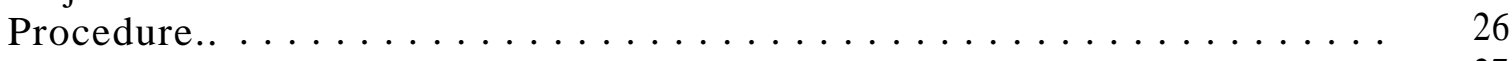

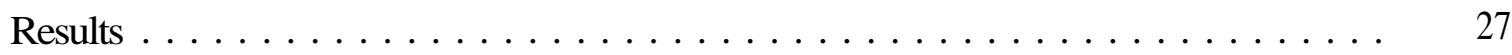

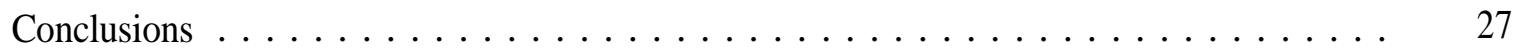

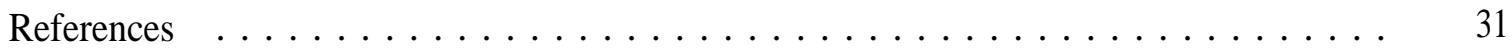

5.0 DESIGN OF FIELD OBSERVATIONS OF TRUCK DRIVERS $\ldots \ldots \ldots \ldots \ldots \ldots$

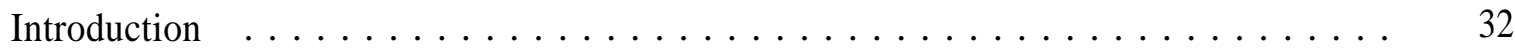

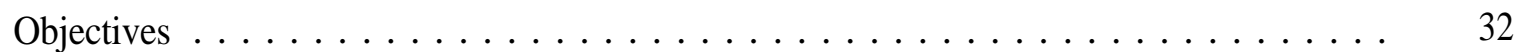

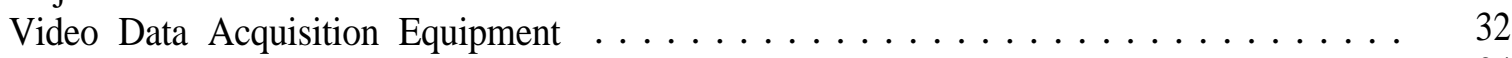

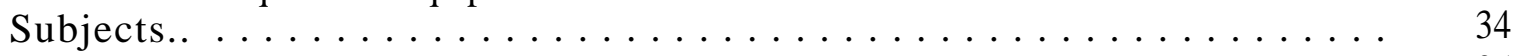

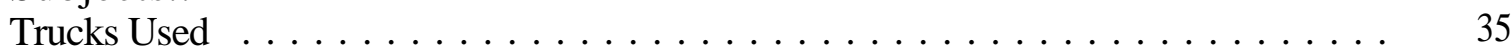

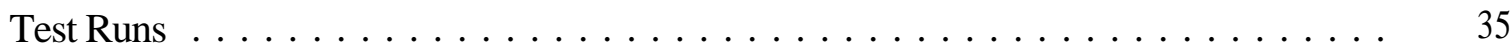

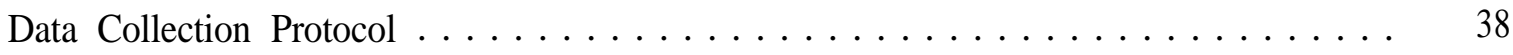




\section{TABLE OF CONTENTS (Continued)}

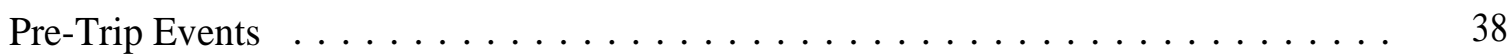

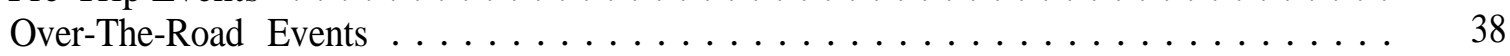

Observe Naturalistic Driving . . . . . . . . . . . . . . . . . . . . . . . . . . . . . . . . . . . . . . . . . . . . .

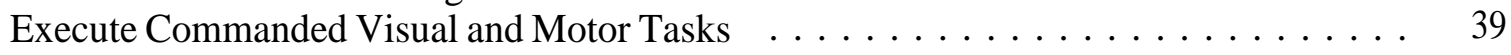

Observe Driver's Hand Activity . . . . . . . . . . . . . . . . . . . . . . 39

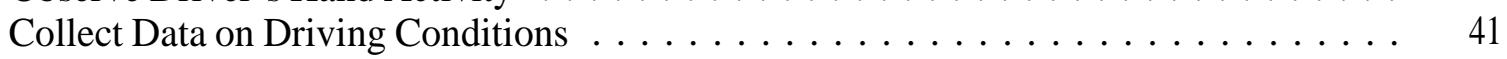

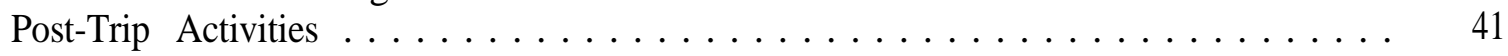

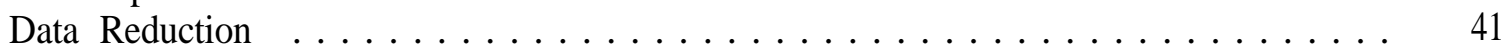

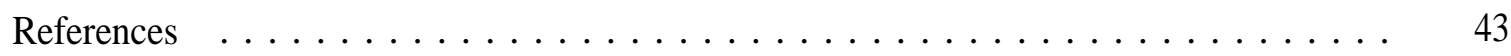

Appendix 5A General Instructions for Test Subjects . . . . . . . . . . . . . . . 44

6.0 RESULTS OF FIELD OBSERVATIONS OF TRUCK DRIVERS $\ldots \ldots \ldots \ldots$

Natural Glance Allocation During Open Road Driving on Rural Freeways . . . . . . . . . 45

Commanded Visual Tasks . . . . . . . . . . . . . . . . . . . 48

Effects of Light Conditions and Traffic Conditions on Natural Glances . . . . . . . . . . 52

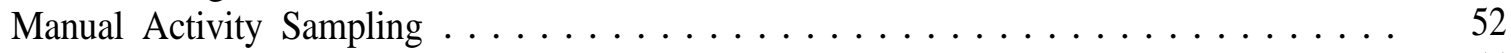

A Sample Characterization of the Driving Conditions in the Data Collection Runs . . . . 55

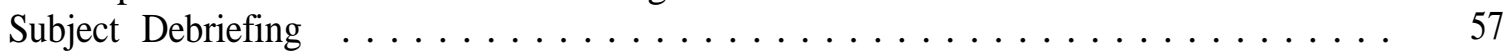

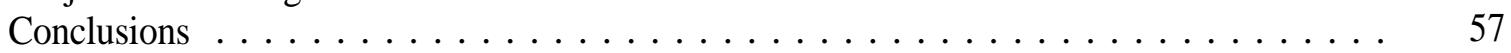

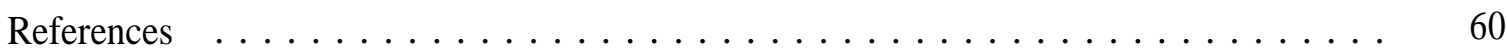

Appendix 6A Characterization of Driving Conditions During the Data

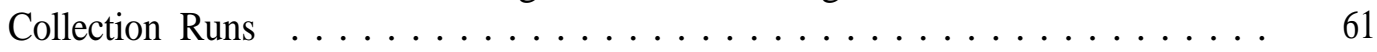

Appendix 6B Truck Driver Workload Study Subject Debriefing Summary . . . . . . . . 63

7.0 PRELIMINARY ANALYSIS OF FEATURES OF IN-CAB DEVICES $\ldots \ldots \ldots \ldots$

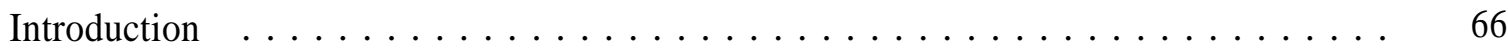

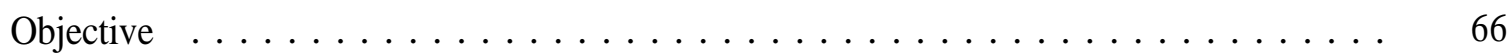

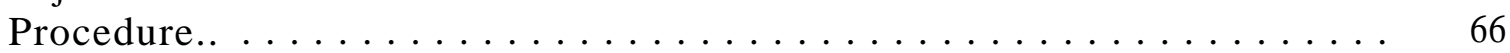

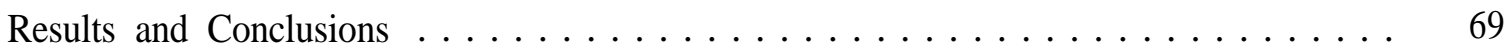

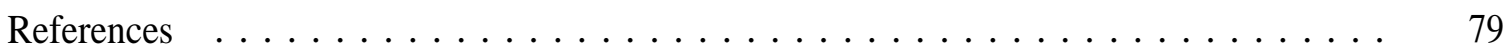

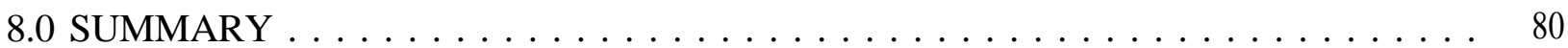


TABLE OF CONTENTS (Continued)

\section{Tables}

Table 3-1 Approximate Relative Importance of Each Factor . . . . . . . . . . . . . . 17

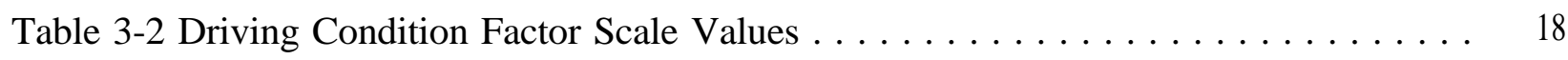

Table 4-1 Truck Driving Tasks $\ldots \ldots \ldots \ldots \ldots \ldots \ldots \ldots \ldots \ldots$

Table 4-2 Difficulty and Importance to Safety Ratings of Driving Tasks Under Three Driving Conditions ........................... 28

Table $5-1$ Subject Biographical Data ..................... 36

Table 5 -2 Summary of Data Collection Runs . . . . . . . . . . . . . . 37

Table 5-3 Left Hand and Right Hand Activity Categories Used In Manual Activity Sampling . . 40

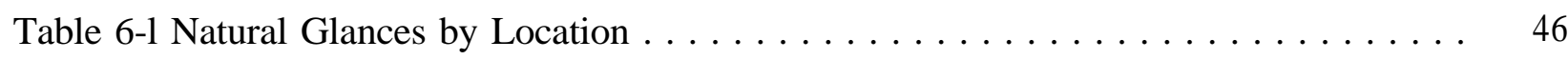

Table 6-2 Illustrative Differences in Driver Visual Allocation . . . . . . . . . . . . . 49

Table 6-3 Protocol for Commanded Tasks $\ldots \ldots \ldots \ldots \ldots \ldots \ldots$

Table 6-4 Summary of Commanded Tasks By Task-All Subjects Combined . . . . . . . . 51

Table 6-5 Natural Glances for Subjects 1, 2, 6 and 8 Combined Nominal Three Minute Segment of Driving Time By Location . . . . . . . . . . . 53

Table 6-6 Illustrative Manual Activity Data Subject 9 Rural Highway Driving . . . . . . . . 54

Table 6-7 Percent of Time Engaged in Hand Activities All Subjects Combined Rural Highway

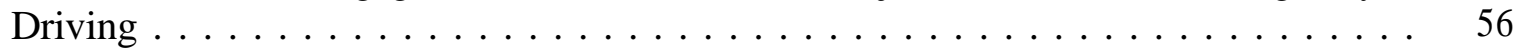

Table 6-8 Allocation of Natural Glances By Road and Off Road Locations Rural HighwayNominal Three Minute Segments of Open Road Driving . . . . . . . . . . . . . 59

Table 7-1 Protocol for NHTSA HV Analysis of In-Cab Device Features . . . . . . . . . . 67

Table 7-2 Voice Communications Systems . . . . . . . . . . . . . . . . . 70

Table 7-3 Vehicle Navigation and Route Guidance Systems . . . . . . . . . . . . . . . 72

Table 7-4 Single/Integrated Displays (Status Displays) . . . . . . . . . . . . . . . 75

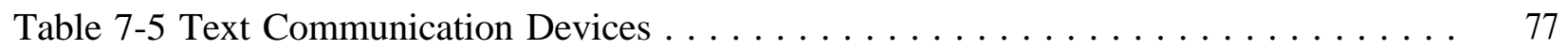




\section{TABLE OF CONTENTS (Continued)}

Figure 3-1 Tradeoff Pairs Evaluated by Heavy Vehicle Drivers . . . . . . . . . . . . . 15

Figure 3-2 Proportion of Drivers Choosing Each Tradeoff Pair . . . . . . . . . . . . 16

Figure 4-1 Difficulty and Importance to Safety Scales $\ldots \ldots \ldots \ldots$

Figure 5-1 Diagram of Video Recording System $\ldots \ldots \ldots \ldots \ldots$ 


\title{
1.0 BACKGROUND
}

\author{
$\underline{\text { Introduction }}$
}

This report provides the results of our task analysis efforts for Task 3 of the NHTSA heavy vehicle workload assessment project. This activity is intended to fill in data gaps that will support the development of the heavy vehicle driver workload assessment protocol. It departs somewhat from the emphasis included in the original Battelle proposal, i.e., almost exclusive on-the-road data collection. These changes, reviewed and approved by the NHTSA COTR, reflect a better understanding of the project requirements and real world limitations associated with on-the-road data collection using commercial drivers on their assigned routes.

\section{Objectives}

The objectives of this data collection effort are as follows:

- $\quad$ Ascertain what the word "workload" means to professional drivers;

- $\quad$ Evaluate the demand placed on the driver by various driving conditions;

- $\quad$ Determine the safety criticality and difficulty of selected standard driving tasks;

- $\quad$ Pilot-test a subset of the data collection equipment that will be used in subsequent tasks on this project;

- $\quad$ Collect on-the-road data on the perceptual, motor, and cognitive loads imposed by various tasks and in-cab behaviors found in trucks today; and

- $\quad$ Begin task analysis of selected high-technology in-cab devices to determine the nature of their interactions with drivers and loads that may be placed on drivers.

\section{Scope}

Data were collected to glean what the term "workload" means to professional drivers. Drivers at a local area truck stop were asked a variety of open-ended questions to prompt discussion of what they consider workload-inducing aspects of their jobs. The results suggest a discrepancy between their interpretation of the term and the level of analysis we seek for in-cab device assessment. Based on the results obtained, a link between the job-level interpretation of workload and the device interactionlevel interpretation of workload is postulated.

The driving demand analysis was approached by means of psychological scaling of assessments provided by professional drivers. Drivers were given pairs of driving conditions developed from various combinations of traffic density, lighting, roadway type, visibility, and traction. They chose which driving condition of each pair was considered the more demanding. Conjoint analysis scaling techniques were then applied to the data and a demand scale was developed. This scale was developed to provide a useful index of the workload imposed by driving conditions outside the cab on the primary task of controlling the vehicle. 
For assessment of driving task difficulty and safety criticality, professional drivers were asked to rate the safety criticality and difficulty of selected driving tasks (maneuvers) under three driving conditions selected to span the range of demand derived from the demand analysis. Originally, relative frequency of tasks was also an object of investigation. However, further reflection suggested that this feature of tasks is highly dependent of the specifics of a particular driver's routes, season of the year, behavior of other vehicles on the road, etc. So, Battelle decided to drop this analysis and the NHTSA COTR concurred with this decision. The safety criticality and difficulty data are useful to the extent that they allow us to prioritize tasks (and tasks within driving conditions) that we wish to include as standard tasks in the protocol.

The on-the-road data collection was comprised of visual allocation data, in-cab manual activity sampling, and driver commentary about the tasks of driving. Naturalistic observations were made of the driver's visual allocation strategies to various visual areas under various driving conditions. Additionally, the ride-along observer asked the driver to visually scan selected gauges on the instrument panel or manipulate instrument panel controls when the driving task permitted. Our instructions emphasized that the driver is the final judge on whether or when to respond. Under no circumstances was the driver asked to perform any action that was not a part of a normal drive. Given that this was a sample of opportunity, Battelle and R \& R Research made best efforts to schedule ride-along observations under a variety of driving conditions. A total of nine drivers were observed for this portion of the work. It should be borne in mind, therefore, that this was a field observational study, not an experiment. We expect to refrain from testing experimental hypotheses until we are in a position to use our own drivers and trucks for specific experimentation. In the Task 3 work, we relied on commercial drivers on their regular routes and so were severely constrained in manipulating any factors of interest to us.

The data collected on the road is useful for determining the range of visual demands imposed by tasks conducted in trucks without high technology devices and how visual allocation varies across drivers and by driving conditions. Similarly, the manual activity sampling method provides a rough indication of the degree to which both hands are dedicated to steering under normal driving without high-technology in-cab devices and individual differences in the allocation of the hands to various incab tasks. This type of data is useful to direct protocol development in terms of procedures, measures, and equipment, as well as providing preliminary baseline data for comparative purposes in analyzing selected in-cab devices.

A preliminary task analysis was completed on selected categories of high-technology in-cab devices. Specifically, classes of in-cab devices were characterized in terms of various loads placed on the driver, the locus of interaction (driver initiated or device initiated), and other related dimensions. This preliminary assessment is valuable in suggesting the kinds of in-cab devices we might wish to use for the evaluation with the to-be-developed protocol. It also suggests classes of measures and phenomena that will guide protocol development. It is imperative that a more detailed cognitive task analysis be conducted on the two to-be-selected devices that will be evaluated with the developed workload assessment protocol. However, at this time such efforts would be unfocussed and costly and so were not pursued. 


\section{$\underline{\text { Report Organization }}$}

Subsequent sections of this report are developed as independent study reports:

- Section 2.0 describes the method, data, and results of our efforts to capture what the term "workload" means to a professional heavy vehicle driver.

- $\quad$ Section 3.0 describes the demand assessment of selected driving conditions.

Section 4.0 reports the approach and results of the driving task safety criticality and difficulty assessments.

- Section 5.0 provides a description of the design of the on-the-road observations.

- Section 6.0 presents the results of the on-the-road data collection.

- Section 7.0 provides a preliminary task analysis on categories of high-technology incab devices.

Section 8.0 summarizes the task analysis effort.

Various appendices are provided as appropriate. 


\subsection{ASCERTAINING THE DIMENSIONS OF TRUCK DRIVER WORKLOAD}

\section{$\underline{\text { Introduction }}$}

The purpose of this research is to establish how truck drivers perceive workload in driving, the factors that contribute to this workload, the various arrangement of these factors that they deem to cause the highest workload conditions, and their strategies for coping with workload. This effort supports the task analysis phase and helps establish baseline conditions for empirical testing in Task 6 .

\section{Objective}

The objective of this study was to use personal interviews of current truck drivers to help define what they perceive to be workload and elicit the factors that contribute the most to workload situations.

\section{$\underline{\text { Subjects }}$}

The seven drivers used in the pretest and the 34 tested in the final interview guide varied on several key biographical variables. The ages of the 41 drivers ranged from 21 to 61 years of age and all but one were males. They averaged 17 years of truck driving experience with a range of one to forty-two years. Forty-four percent were independent drivers. Most were nonunion drivers. Forty-four percent received informal training, 38 percent received formal training and 18 percent received no prior training.

Two-thirds drove conventional tractors and one-third drove cabovers. Many tractor makes were evident but Freightliner and Peterbilt accounted for 44 percent of the total. Their tractor equipment averaged 4.5 years in age, ranging from new tractors to ones 14 years of age. Ninety-eight percent hauled only one trailer. Seventy-one percent were on cross-country, long-hauls and 29 percent were regional long-hauls. Two-thirds of the participants hauled dry freight.

All drivers interviewed had CB radios and 98 percent had AM/FM stereo radios. Fifteen percent had satellite tracking systems and 12 percent had cellular telephones.

\section{Procedure}

A personal interview guide was used to achieve the objectives of this study. Drivers were recruited at truck stops to discuss "workload" with members of the research team. The interviews were conducted one on one. The drivers were not paid for their participation. Each interview lasted from 12 to 20 minutes.

Seven drivers were pilot tested to help develop the final interview guide. In the pretest, drivers were asked to describe what workload meant to them. Many responded that workload was part of the job. Others suggested that workload was essentially time constrained stress, i.e., any situation before or during the run to cause them to "run late" was considered workload. Discussions suggested that two 
conditions contributed to "driving" workload: a) increased attentional concentration and b) increased visual, cognitive, and psychomotor activity.

\section{$\underline{\text { Results }}$}

The results of the interviews are tabulated in Appendix 2A.

The interview began with a proposal for defining high workload, namely situations requiring increased activity and concentration. The drivers were then asked to develop examples of their own that produced high workload (Item la). The most common suggestions were: city driving in traffic (11 of 34), heavy traffic (10 of 34), operation in construction zones (7 of 34), and bad weather (4 of $34)$.

The drivers were then asked what made their reported situation a condition of high workload (Item lb). The most common factor involved some aspects of heavy traffic.

The drivers were then asked how they coped with this workload (Item lc). One-third reported paying more attention. Others reported increased mirror sampling and others reduced speed to provide more time to react to the traffic.

At this point, an example of a workload situation developed from the pretest data was offered to get driver reaction. The first involved I-294 around Chicago in rush hour traffic. The driver was asked if he agreed this was a high workload situation and why (Item 2a)? Twenty-one of the 34 drivers agreed this situation involved high workload. They cited high traffic density and unpredictable passenger car behavior as specific causes. Thirteen did not agree that this was a high workload situation. Many felt that other drivers probably said so because of traffic and the fact that people are in a hurry at rush hour, while isolated responses suggested other factors such as the need for trucks on I-294 to frequently change lanes within the dense traffic.

When asked to rate the Chicago situation on a workload scale of 1-10 (Item 2b), 17 of the 21 drivers who considered this a workload situation rated it eight or higher. Drivers were then asked how they compensate for rush hour traffic (Item 2c). Again concentration, reduced speed, and increased mirror sampling were reported.

A second workload situation was offered to drivers for their reaction, namely night driving on an icecovered, rural freeway (Item 3a). Twenty-five of the drivers interviewed did not believe this was high workload. They speculated that drivers who did think of this as high workload are probably not familiar with the situation. Compensatory behavior reported included reduced speed and increased attention.

Item 4 shifted emphasis to truck driver tasks and their effect on workload. Eight tasks were ranked on workload. The mean response rank and its standard deviation for each task are given in Appendix 2A. Note that operation in a construction zone ranked the highest (7.19). This was followed by turning at intersections and curve negotiation.

The next question (Item 5a) was an open-ended one that solicited any other high workload conditions experienced by these drivers. Twenty-eight scenarios were mentioned covering 20 different 
conditions. Three drivers reported truck unloading (not driving related) and backing into blind spots (also not en route driving related). There was a large range of responses to this question from hauling hazardous materials and visibility restrictions to mountainous operations.

When asked about compensatory behavior in these situations (Item 5b), slowing down and paying more attention accounted for 13 of the 20 responses.

\section{$\underline{\text { Conclusions }}$}

Interviews with the seven pretest drivers and the 34 drivers in the final sample offer interesting insights into the workload phenomena in truck driving. Of the five basic dimensions reported (driver factors, truck factors, traffic factors, roadway factors and environmental factors), it was clear that traffic and the environment contribute the most to perceived high driving workload. Truck factors were rarely mentioned, probably because drivers adapt to their rigs and often use the same rig each day.

Drivers often identified workload as stress. In discussion with drivers, the most commonly reported source of stress is being delayed from either driving situations (delays from traffic, construction, accidents and weather), or from non-driving factors beyond their control (shipping, consignee delays, DOT inspections, delays at scales, late load arrivals etc.). Traffic, inclement weather and road construction all lead to increased driving times and delays in making schedules, or increased driving activity to make schedule.

From the interviews one can postulate the following hypothesis. Truck driver workload increases with increased driving activity or increased time stress. If a driver becomes delayed en route (due to weather, traffic, road construction, shipper/consignee delays, etc.) and the delivery schedule becomes jeopardized, then the driver is faced with two no-win trade-off strategies. The driver can try to make up the "lost time" (i.e., drive faster, accept shorter headways, execute more frequent lane changes, etc.) and thereby increase the required driving activity and concentration. Alternatively, the driver can reduce the driving workload in traffic or weather by lowering speed, holding lane position and increasing headways and thereby accept the increased overall stress caused by the time delays. In either case, delays might prompt increased in-cab transactions at inopportune times, e.g. frequent status updates to a dispatcher. Thus, when drivers are running late there appear to be few workload management options available since the alternatives may ultimately result in increased stress levels.

The message for future baseline studies is clear. Traffic conditions, weather conditions, and special conditions (such as construction zones) must be established. Driver coping strategies with these conditions must be measured, especially visual search and speed reductions. Some way of inducing time stress will make the baseline studies more realistic. 


\section{Appendix 2A}

\section{Truck Driver Interview Guide and Tabulation of Responses Truck Driver Interview Summaries}

la. If we assume that high workload is associated with a) increased activity, e.g., mirror use, braking, accelerating, land changing and/or b) increased concentration on the driving task, can you think of any personal driving situations that involved high workload? $(\mathbf{n}=34)$ (Numbers don't add to 34 because of multiple responses)

Confusing signs

City driving in traffic

4-wheeler traffic

Heavy traffic

Driving through mountains

Construction zone

Unloading trailers

Satelite tracking systems use

Highway patrol involvement

Use of scales

Dispatcher interaction

D.O.T. Checks

Bad weather driving

Backing in blind spots

Pot holes

No response

Making time

lb. What exactly is it about this situation that makes it high workload? Probe for driver, environmental, roadway, traffic and truck factors. $(n=34)$

Heavy traffic

Generally causes stress

Careless drivers

Shifting gears

Shifting loads (mountains)

Merging traffic

Braking

Have to concentrate more

D.O.T. harassment

Cars - traffic

Narrow lanes

Waiting

Uncertainty of the situation 
lc. Do you do anything special to compensate for the higher workload when driving in this situation?

Other driver helps navigate $\quad 1$

Avoid situation (travel at night or different road) 2

Pay more attention to driving 11

Drive according to conditions 1

Watch mirrors $\quad 5$

Slow down 6

Think ahead 3

Listen to music 3

Give more stopping distance 2

Don't rush 2

Relax 1

2. Earlier in our study we talked to some drivers who identified several situations they felt involved high workload while driving. I'd like to get your reaction to a couple of these.

One situation involved driving in heavy traffic on a multi-lane freeway in a city. For example, one driver mentioned driving around Chicago on the I-294 tollway in rush hour.

2a. Would this situation represent high workload for you? What exactly is it about this situation that makes it high workload? Probe for driver, environmental, roadway, traffic and truck factors. [If driver feels this is not a high workload situation, ask him to speculate as to why it might be high workload for others.] $(n=34)$

No - other drivers might say so because of:

Heavy traffic

Many lane changes

4-wheeler traffic

Scared of the situation 4

Not skilled drivers $\quad 2$

Cars unpredictable 1

People in a hurry $\quad 4$

Yes, because of:

Heavy traffic (cars) $\quad 8$

Alert -have to watch other people 7

High speeds 1

Being cut-off by cars 1

Bad roads/not marked very well 1

People in a hurry/not careful 1

Low bridges (13'7') 1

Stopping - toll booth 1 
Cars unpredictable (can't stop on a dime)

Change lanes

Increased shifting

2b. On a scale of 1 to 10 , where 10 represents the most extreme case of high workload, how would you rate this situation? (Drivers who said "yes")

$($ standard deviation $=1.35$, mean $=9.17, \mathrm{n}=17$ )

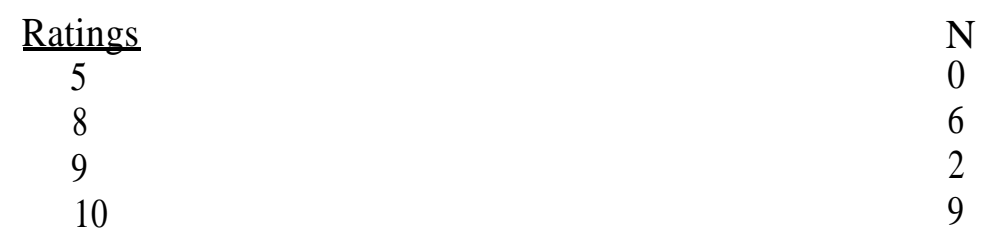

2c. Do you do anything special to compensate for the higher workload when driving in this situation?

Help navigate

Drive according to conditions

Slow down

Watch mirrors

$\mathrm{N}$

Relax

Take a break

Pay more attention

Give more breaking room

3. A second situation mentioned by other drivers was driving at night on an ice-covered, wideopen rural freeway with no traffic.

3a. Would this situation represent high workload for you? What exactly is it about this situation that makes it high workload? Probe for driver, environmental, roadway, traffic and truck factors. [If driver feels this is not a high workload situation, ask him to speculate as to why it might be high workload for others.] $(n=34)$

No, others might say yes because:

They are not familiar to the condition $\quad 16$

They don't respect the condition 1

Drive to fast 2

Behind schedule/in a hurry 2

Can't predict other's reactions 1

Conditions unpredictable 2

Visibility 
Yes, because of:

$\begin{array}{ll}\text { Covered bridges } & 1 \\ \text { Stressful } & 2 \\ \text { Unpredictable conditions } & 4 \\ \text { Headlights glare } & 1 \\ \text { Visibility } & 1\end{array}$

3b. Do you do anything special to compensate for the higher workload when driving in this situation?

\section{Slow down}

4

1

1

Pay more attention

Drive according to the conditions 2

Down shift

Don't rush

Watch mirrors

Maintain safe following distance

4. Next, I'm going to show you a list of eight common tasks that truck drivers regularly perform while driving. I would like you to rank these from " 1 " to " 8 " in order of increasing workload. For this purpose, a "1" means the task has the lowest workload, while an "8" means the task has the highest workload. $(n=21)$

$\begin{array}{cr}\text { Mean } & \text { Std. De } \\ & \\ 2.33 & 1.35 \\ 2.42 & 1.94 \\ 3.57 & 1.53 \\ 4.24 & 1.48 \\ 4.48 & 2.20 \\ 5.14 & 1.68 \\ 6.62 & 1.24 \\ 7.19 & 1.25\end{array}$

\section{Command}

Check your mirrors

Eat or smoke while driving

Change lanes

Pass another vehicle on the left

Enter a freeway

Negotiate a curve and stay in your lane

Make a turn at an intersection

Driving through a construction zone

5a. Can you think of any driving situations we HAVE NOT mentioned that involve high workload for you? Tell me about them. What exactly is it about this situation that makes it high workload? Probe for driver, environmental, roadway, traffic and truck factors. $(n=34)$

Construction zone

Hazardous material

D.O.T. scales

Mountains

Timepressure multiple drops

City traffic - rush hour 
Flat-bed - chaining things down

Cars - drunks

Serious accidents

Visibility restrictions

Cars don't respect big loads

Unpredictable 4-wheeler traffic

Highway patrol

Worn tread

New drivers

D.O.T.

Customs(U.S.-Canadian)

Unloading trucks

Backing in blind spots

Rain

5b. Do you do anything special to compensate for the higher workload when driving in this situation?

Pre-plan trip

Slow down - don't rush

Pay more attention

Relax

Avoid the situation

Rest

Give enough stopping distance 


\subsection{ASSESSMENT OF DRIVING CONDITION DEMAND}

\section{Introduction}

The individual driver's own subjective assessment of driving demands is an important component for understanding the demands placed on heavy vehicle operators and the impact that these demands put on actual driving behavior and performance. It is important to know and to measure what driving conditions heavy vehicle operators subjectively perceive or evaluate as being low, moderate, or high in driver workload. It is important to do this by using a reliable, sensitive, and valid quantitative measure of the subjective demand or workload a driver experiences under different driving conditions.

Such a measure would allow us to begin to assess how the levels of different environmental factors and/or vehicle-related factors might tradeoff under different conditions to either increase or reduce driver demand. It would also allow us to assess the degree to which individual differences among heavy vehicle operators contribute to the perception of high or low workload conditions. That is, it would be useful to know if certain factors, or combinations of factors, are considered by almost all operators to produce high driver demand or to be a threat to operational safety while other factors may be perceived as high or low depending on individual differences variables such as age or experience. This study was designed to provide some initial data to assess these issues and to show that a powerful scaling technique, conjoint analysis (Nygren, 1985a, 1985b, 1986), can be used to obtain a valid and reliable measure for assessing driving condition demand.

Conjoint analysis is a multidimensional or multi-factor scaling technique that numerically scales the joint effects or combinations of the levels of several independent factors (e.g., driving condition factors such as lighting levels, traffic density, and roadway type) on a dependent variable (judged driving demand). It is mathematically similar to regression techniques. As explained in Boff and Lincoln (1988), in conjoint scaling, two (or more) aspects or factors of a situation are placed in a matrix. Each factor has a number of levels. The combination of each level of each factor with the other(s) is then ranked. For example, in a $2 \times 2$ matrix of lighting conditions vs. traffic density (day vs. night, light vs. heavy), judged demand is ranked for each combination from 1 (least demand) to 4 (most demand). Next, agreement among the rankings by the group of judges (e.g., drivers) is tested to determine if a common-model is appropriate. If agreement is high, a common rank-order based on the mean rank-order for all the subjects is obtained. This is then rescaled using conjoint scaling techniques to yield an interval-level scales of value for the combination of the factors (i.e., the values are not just rank orders but real numbers with meaningful intervals between them). In this way, multiple factors define conditions which are scaled onto a unidimensional scale of driving demand. Additional technical details are included in Appendix 3A of this section.

\section{$\underline{\text { Objective }}$}

The goal of this study was to use conjoint analysis to produce a unidimensional scaled measure of perceived demand under different driving conditions including traffic density (heavy vs. light), lighting (day vs. night), roadway type (undivided vs. divided), visibility (good vs. poor), and traction (good vs. poor). This produced a $2 \times 2 \times 2 \times 2 \times 2$ design of 32 driving condition combinations. Three widely used data collection procedures were possible for this study. One the "full rank sorting" 
technique in which the subject rank orders all possible stimulus combinations from lowest to highest on the subjective variable (e.g., workload). Although full sorts have the advantage of providing complete data for the maximum number of tests of the independence properties mentioned above, a complete ranking of all stimuli can be a very demanding task for even moderate stimulus designs. Evaluating the 32 combinations from the five-factor $2 \times 2 \times 2 \times 2 \times 2$ design used here would be extremely difficult for most subjects to do reliably. A second data collection procedure, obtaining a complete set of paired comparisons of all factor combinations, would be far richer than the full-sort ranking data. However, the number of paired comparisons for the 32 stimuli used here is again far too large $(n=496)$ to be feasible in this study.

A "tradeoff matrix" procedure provides the best practical approach for collecting conjoint data in this kind of study. Only two factors are evaluated at a time, and combinations of the pair of factors are ranked. Although this reduces the cognitive demands placed on the individual rater, the capability of doing a full evaluation of the independence properties is lost. However, the data do support sufficient testing of simple independence of the factors. This allows us to evaluate the quality of an additive representation. For these reasons the tradeoff procedure was chosen.

\section{$\underline{\text { Subjects }}$}

Fifty-five heavy vehicle operators were recruited to participate in this study from a truck stop in the Columbus area. As such, they represent a sample of opportunity.

\section{Procedure}

Data were collected from each heavy vehicle operator separately. The data collector first explained the meaning of the terms "driving conditions" and "demand", i.e.,:

- $\quad$ DRIVING CONDITIONS on the road involve, among other things, traffic density (heavy vs. light), lighting (day vs. night), roadway type (undivided vs. divided), visibility (good vs. poor) and traction (good vs. poor).

- $\quad$ People commonly think of the DEMAND of on-the-road driving conditions in terms of how much the driver must focus his attention on the driving situation to safely control the vehicle. The greater the DEMAND, the less "forgiving" the driving conditions are of lapses in attention.

Ten tradeoff pairs were evaluated by each driver (see Figure 3-1). The drivers indicated which driving condition was the most demanding of the pair.

Each pair of factors, together with its complementary pair, produces a $2 \times 2$ matrix of factor combinations, and these four cells can be ranked from least to most demanding for the driver. Because all five factors are defined to be clearly ordinal in that there is an unambiguously low and high level for each factor as it relates to driver demand (e.g., heavy traffic vs. light traffic), the lowest and highest ranked cells in each $2 \times 2$ matrix are already known to be ' 1 ' and ' 4 ' respectively. 
The task was reduced, then, to a simple tradeoff paired comparison procedure to determine the second and third most demanding combination. For example, pair 1 from Figure 3-1 leads to the following $2 \times 2$ table:

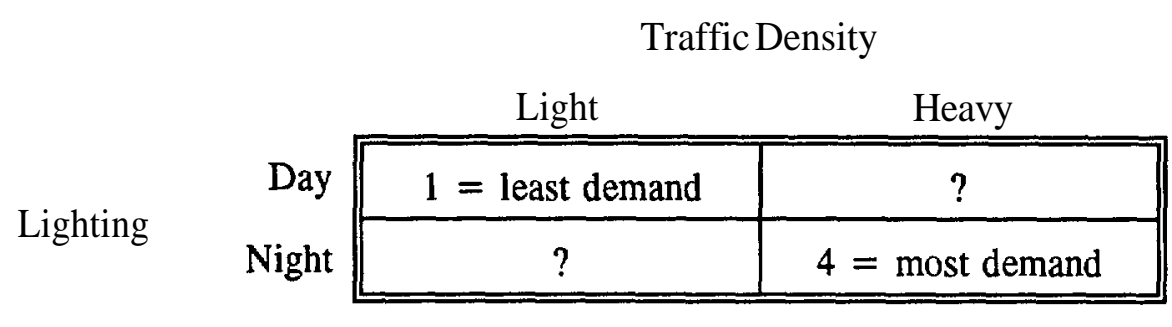

For Lighting vs. Traffic Density, the critical comparison for each driver then becomes "which of these is more demanding -- nighttime with light traffic or daylight with heavy traffic?" This was the task the drivers were asked to do for each of the ten pairs of factors. The ten $2 \times 2$ rank orders of 1-4 were then formed and the data submitted to the CMSCAL program for conjoint scaling (Nygren, 1986).

\section{$\underline{\text { Results }}$}

Figure 3-2 presents the results of the 10 tradeoff paired comparisons. Except for two of the pairs, the agreement among drivers is very strong, with proportions in the .80 to 1. OO range. All 10 tradeoff proportions were found to be significantly different from an equal or 50-50 split $(\mathrm{p}<.05$ in each case). These results suggest rather strong consensus among the drivers as to what factors and factor levels produce the greatest demand.

The conjoint scaling analysis provides some further evidence for this consensus. Table 3-1 shows the relative importance of each of the five factors in determining the overall demand level of the 32 combinations. It is interesting that the two most important factors, traction and visibility, are environmental factors that cannot be controlled by the driver. The other three factors, traffic density, highway type, and lighting, can be controlled, at least to some extent, by the driver. In fact, one might argue that the ordering of perceived demand is the reverse of the ordering of perceived control over the driving conditions. This is an inference only, however, and drivers were not asked about this.

Table 3-2 shows the final estimated demand scale values for each of the 32 driving conditions. The measure, because it has interval properties, can be rescaled without loss of generality. For convenience it is scaled so that the lowest demand combination (good traction, good visibility, low density, divided highway, and daylight) is given a value of 0.0 and the highest (poor traction, poor visibility, high density, undivided highway, and night) is given a value of 100 . Scale values for the remaining 30 combinations can be easily obtained. Note, however, that although differences among the 32 scale values are quite meaningful, the lowest combination is not assumed to have a true zero demand or workload level associated with it. 
Figure 3-1

Tradeoff Pairs Evaluated by Heavy Vehicle Drivers

1. Nighttime with Light Traffic

2. Nighttime on a Divided Highway

3. Good Visibility at Nighttime

4. Good Traction at Nighttime

5. Heavy Traffic on a Divided Highway

6. Good Visibility and Heavy Traffic

7. Undivided Highway and Good Visibility

8. Heavy Traffic and Good Traction

9. Poor Visibility and Good Traction

10. Undivided Highway and Good Traction or Daylight with Heavy Traffic

or Daylight on an Undivided Highway

or Poor Visibility in Daylight

or Poor Traction in Daylight

or Light Traffic on an Undivided Highway

or Poor Visibility and Light Traffic

or Divided Highway and Poor Visibility

or Light Traffic and Poor Traction

or Good Visibility and Poor Traction

or Divided Highway and Poor Traction 
Figure 3-2

\section{Proportion of Drivers Choosing Each Tradeoff Pair}

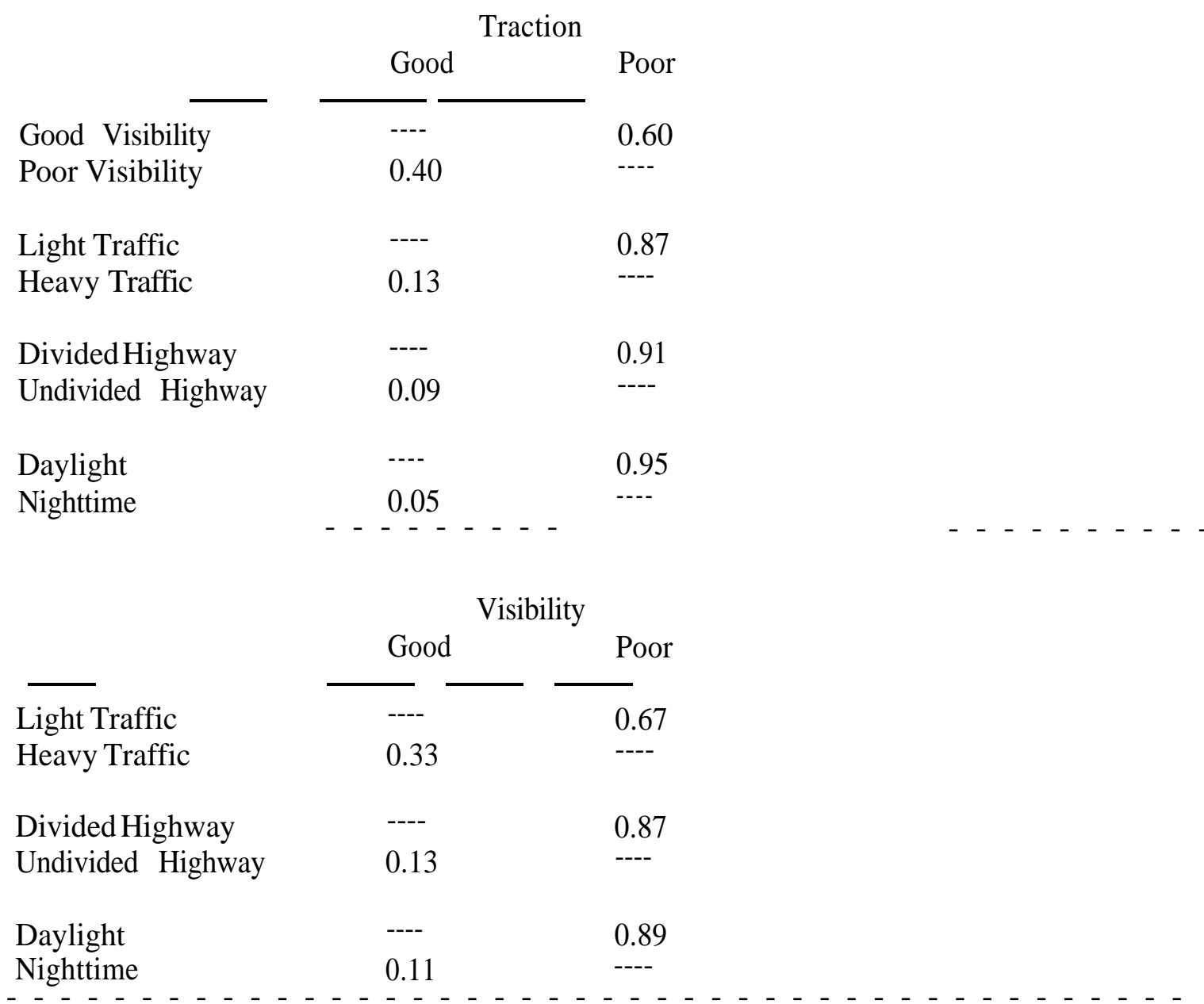

\begin{tabular}{|c|c|c|}
\hline & \multicolumn{2}{|c|}{ Traffic } \\
\hline & Light & Heavy \\
\hline DividedHighway & $\cdots$ & 0.80 \\
\hline Undivided Highway & 0.20 & ---- \\
\hline Daylight & --- & 0.89 \\
\hline Nighttime & 0.11 & ---- \\
\hline
\end{tabular}

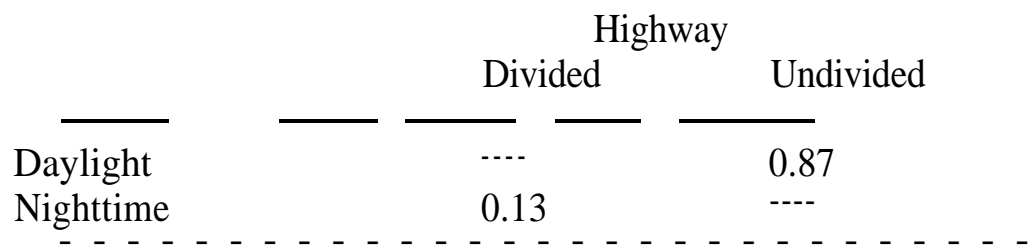

Note: Proportions in each matrix are based on paired comparison data across all 55 subjects, 
Table 3-1

\title{
Approximate Relative Importance of Each Factor
}

\author{
$51.61 \%$ for factor A - Traction - Good/Poor. \\ $25.82 \%$ for factor B - Visibility - Good/Poor. \\ $12.90 \%$ for factor C - Traffic Density - Light/Heavy. \\ $6.45 \%$ for factor D - Highway - Divided/Undivided. \\ $3.22 \%$ for factor E - Lighting - Day/Night.
}

Traction $>$ Visibility $>$ Trafflc Density $>$ Highway Type $>$ Lighting,

A note about the discrepancy between these results and the results for Item $3 \mathrm{a}$ of Section 2.0 is in order. Ice-covered roads were not considered to cause high workload in that assessment, yet that factor was the main determiner of driving demand. One possibility for the discrepancy is that the former assessment reflects an attitude of confidence common among drivers, pilots, and other professionals. The demand scaling used relative judgments rather than absolute judgments and might be considered more sensitive in that regard. It is also possible that workload and demand are not synonymous though they are positively correlated in the real world.

\section{Conclusions}

The demand assessment indicates that drivers perceive poor traction and poor visibility to create the most demanding driving. Heavy traffic density ranks third in influencing driving demand. This suggests that the factors that most influence driving demand are those in which the driver has the least control over the vehicle. Drivers can sometimes minimize this demand by scheduling departures to avoid traffic, by slowing down on slippery roads, and by avoiding close car following, among other tactics.

The results of this study are encouraging because they indicate a rather strong consensus among drivers as to which factors lead to greatest demand. In addition, the data, as averaged across subjects, was perfectly compatible with an additive representation. No violations of independence were found across the five factors. Even at the individual subject level, all subjects' data fit an additive representation perfectly or with at most two independence violations. Although the intent of this study was not to show the predictive validity of the demand scale obtained from the conjoint analysis, the results are very encouraging and the estimated scale values for the 32 combinations could be used to provide the basis for such a study.

These results have several implications for protocol development:

To create high demand of the driving task, traction, visibility and traffic density should be manipulated;

- $\quad$ These factors should be considered in simulator study design when feasible; 
Table 3-2

Driving Condition Factor Scale Values

\begin{tabular}{|c|c|c|c|c|c|c|c|}
\hline Stimulus Levels* & A & B & $\mathrm{C}$ & $\mathrm{D}$ & $\mathrm{E}$ & Standard & Rescaled \\
\hline 1 & 1 & 1 & 1 & 1 & 1 & -3.753 & 0.000 \\
\hline 2 & 1 & 1 & 1 & 1 & 2 & -3.511 & 3.224 \\
\hline 3 & 1 & 1 & 1 & 2 & 1 & -3.269 & 6.448 \\
\hline 4 & 1 & 1 & 1 & 2 & 2 & -3.027 & 9.672 \\
\hline 5 & 1 & 1 & 2 & 1 & 1 & -2.785 & 12.896 \\
\hline 6 & 1 & 1 & 2 & 1 & 2 & -2.543 & 16.120 \\
\hline 7 & 1 & 1 & 2 & 2 & 1 & -2.301 & 19.345 \\
\hline 8 & 1 & 1 & 2 & 2 & 2 & -2.059 & 22.569 \\
\hline 9 & 1 & 2 & 1 & 1 & 1 & -1.815 & 25.819 \\
\hline 10 & 1 & 2 & 2 & 2 & 1 & -1.573 & 29.043 \\
\hline 11 & 1 & 2 & 1 & 2 & 1 & -1.331 & 32.268 \\
\hline 12 & 1 & 2 & 1 & 2 & 2 & -1.089 & 35.492 \\
\hline 13 & 1 & 2 & 2 & 1 & 1 & -0.847 & 38.716 \\
\hline 14 & 1 & 2 & 2 & 1 & 2 & -0.605 & 41.940 \\
\hline 15 & 1 & 2 & 2 & 2 & 1 & -0.363 & 45.164 \\
\hline 16 & 1 & 2 & 2 & 2 & 2 & -0.121 & 48.388 \\
\hline 17 & 2 & 1 & 1 & 1 & 1 & 0.121 & 51.612 \\
\hline 18 & 2 & 1 & 1 & 1 & 2 & 0.363 & 54.836 \\
\hline 19 & 2 & 1 & 1 & 2 & 1 & 0.605 & 58.060 \\
\hline 20 & 2 & 1 & 1 & 2 & 2 & 0.847 & 61.284 \\
\hline 21 & 2 & 1 & 2 & 1 & 1 & 1.089 & 64.508 \\
\hline 22 & 2 & 1 & 2 & 1 & 2 & 1.331 & 67.732 \\
\hline 23 & 2 & 1 & 2 & 2 & 1 & 1.573 & 70.957 \\
\hline 24 & 2 & 1 & 2 & 2 & 2 & 1.815 & 74.181 \\
\hline 25 & 2 & 2 & 1 & 1 & 1 & 2.059 & 77.431 \\
\hline 26 & 2 & 2 & 1 & 1 & 2 & 2.301 & 80.655 \\
\hline 27 & 2 & 2 & 1 & 2 & 1 & 2.543 & 83.880 \\
\hline 28 & 2 & 2 & 1 & 2 & 2 & 2.785 & 87.104 \\
\hline 29 & 2 & 2 & 2 & 1 & 1 & 3.027 & 90.328 \\
\hline 30 & 2 & 2 & 2 & 1 & 2 & 3.269 & 93.552 \\
\hline 31 & 2 & 2 & 2 & 2 & 1 & 3.511 & 96.776 \\
\hline 32 & 2 & 2 & 2 & 2 & 2 & 3.753 & 100.000 \\
\hline
\end{tabular}

\footnotetext{
*A $=$ Traction

$\mathrm{B}=$ Visibility

C $=$ Traffic Density

$\mathrm{D}=$ Highway Type

$\mathrm{E}=$ Day/Night
} 
- $\quad$ Since traction, visibility, and traffic effects are likely to be difficult to emulate fully in a simulator, appropriately scheduled on-the-road data collection is imperative; and

- $\quad$ By way of in-cab device use, systems likely to be used in inclement weather (e.g. InVehicle Safety Advisory and Warning Systems (IVSAWS)) must be evaluated in appropriately stringent conditions. 


\section{References}

(1) Boff, K. R. \& Lincoln, J. E. (1988). Engineering data compendium: Human nercention and performance (Volumes I - III). Wright-Patterson AFB, OH: Armstrong Aerospace Medical Research Laboratory (AAMRL).

(1) Nygren, T. E. (1985a). An examination of conditional violations of axioms for additive conjoint measurement. Applied Psychological Measurement, 9, 249-264.

(2) Nygren, T. E. (1985b). Axiomatic and numeric conjoint measurement: A comparison of three methods for obtaining subjective workload (SWAT) rankings. Proceedings of the IEEE 1985 NAECON Conference, 2, 878-883.

(3) Nygren, T. E. (1986). A two stage algorithm for assessing violations of additivity via axiomatic and numerical conjoint analysis. Psvchometrika, 51, 483-491.

(4) Reid, G. B. \& Nygren, T. E. (1988). Mental workload assessment techniques. In P. Hancock and N. Meshkati (Eds.), Human mental workload. Amsterdam, The Netherlands: Elsevier. 


\section{Appendix 3A}

\section{Further Description of Conjoint Analysis}

Conjoint analysis is a multidimensional or multi-factor scaling technique that numerically scales the joint effects or combinations of the levels of several independent factors on a dependent variable. It is mathematically very similar to ANOVA and regression techniques with one very important distinction. Conjoint analysis treats the dependent measure as though it is only order-preserving (i.e., an ordinal scale) and attempts to monotonically transform the dependent variable in such a way that a simple additive model, or, equivalently in ANOVA terms, a main effects model with no interaction terms, will explain as much variance in the data as possible. Thus, the net effect is to simultaneously re-scale both the independent and dependent variables so that a psychological judgment model suggesting an additive combination rule for the relevant factors best fits the data.

For example, the Subjective Workload Assessment Technique (SWAT) methodology for assessing mental workload (Reid and Nygren, 1988) is based on conjoint analysis methodology. In SWAT three dimensions of perceived workload are hypothesized (time load, mental effort load, and psychological stress load) each with three levels (low, medium, and high) for a total of 27 workload combinations that can be ranked by a rater from least to most workload. Conjoint scaling is then used to monotonically transform the ranks of ' 1 ' to ' 27 ' to form an additive rescaling of the ranking data so as to fit a three-factor additive model of perceived mental workload. The end result is that an event scored, for example, as a 2-1-3 (moderate time demands, low mental effort demands, high stress demands) can be transformed into an interval-scaled workload value (although many researchers believe that behavioral data will often not meet the assumptions required for interval scaling). The real advantage of this procedure over other scaling procedures like Thurstone scaling is that it can be done reliably at the individual rater as well as group level; a large number of replications across or within individuals is not required.

In addition to providing a rescaling of the data and a measure of the overall fit of the monotonically transformed data to an additive factor model, conjoint analysis allows one to at least partially evaluate the validity of the additive combination rule as the psychological model by which individuals are assumed to combine the multidimensional information. Two independence properties of additive representations must hold if the model is a valid one. Other properties are necessary for an additive representation, but they have beenfound to be both difficult to assess empirically and only weakly diagnostic (Nygren, 1985).

The first of the two independence properties is simple independence, which asserts that the factors are psychologically independent of one another. That is, the perceived ordering of the levels of one factor must remain the same regardless of the levels of any other factor. Thus, if driving during daylight is perceived to be less demanding than driving at night, then this ordering should be the same regardless of what other combinations of factors like visibility level or traction combine with it.

A second and similar form of independence extends this idea. The property, known as joint independence, states that the ordering of the combined effects of two factors must be preserved across levels of the other factors. Thus, if driving during daylight with heavy traffic is perceived as being more demanding than driving at night with light traffic, this ordering should be the same regardless of levels of other factors like visibility level or traction. 
Consideration of these independence properties forces a vital practical methodological constraint in many applications of conjoint analysis. It is: How can we best obtain the critical data needed to both test the assumptions of the additive conjoint model and obtain a stable and reliable additive scaling solution? This methodological consideration often involves a tradeoff. It is important to have a conjoint data collection method that is rich enough to examine the simple independence property; yet, at the same time, to minimize the complexity of the judgment task because of the complex nature of the multidimensional stimuli being judged. Finally, the data collection procedure must be complete enough to provide a means of assessing individual differences on the subjective workload scale. 


\subsection{DIFFICULTY AND IMPORTANCE RATINGS OF TRUCK DRIVING TASKS UNDER VARYING DRIVING CONDITIONS}

\section{Introduction}

The evaluation of in-cab devices will require the use of representative truck driving tasks which are highly critical to safety, or are difficult to execute, in order to place performance demand on the truck driver. The selection of this sample of driving tasks required that we determine which driving tasks were more difficult than others and which were more important to safety than others. We also considered driving conditions because this parameter can affect the difficulty and importance of a driving task. Thus, the purpose of this study was to determine the difficulty and importance to safety of a set of truck driving tasks under varying driving conditions.

Four efforts were required to perform this study: identification of a set of varying driving conditions, determining a list of truck driving tasks, creation of judgement scales for difficulty and importance to safety, preparation of a set of instructions, and identification of a group of drivers. The approach we took to these efforts is discussed below.

As reported in Section 3.0, the relative importance of selected factors in driving conditions was determined. These factors, listed in order of importance, were: traction, visibility, traffic density, divided vs undivided highway, and daylight vs nighttime. The relative importance of all combinations of these factors was determined and ranked (see Table 3-2).

For this study, three conditions were selected from this list: one representing the best set of conditions, one representing one of the worst set of conditions and one representing conditions in between. The first set of conditions (Condition 1: good traction, good visibility, low traffic density, divided highway, daylight) was considered typical and the best driving condition. The second set of conditions (Condition 2: good traction, poor visibility, heavy traffic, undivided highway, nighttime) was selected because it ranked in the middle of the rankings (48.388, see Table 3-2), but was considered a very degraded set of conditions. The third set of conditions (Condition 3: poor traction, poor visibility, heavy traffic, divided highway, daylight) was considered realistic and one of the worse set of conditions. The very worse set of conditions (poor traction, poor visibility, heavy traffic, undivided highway, nighttime) was not selected. Based on discussions with drivers, it was hypothesized that no one would drive in these extreme conditions.

In the Task 2 Interim Report for this effort, a list of truck driving tasks was identified from a variety of task analyses. A set of 30 driving tasks was selected from this list for this study (see Table 4-1). Those tasks not selected, such as "shift gears", "glance at gauges", "start vehicle", were considered driving actions and we were more interested in the difficulty and importance to safety of complex driving tasks than the simple actions which make up the tasks.

For truck drivers to rank the tasks in difficulty and importance to safety, two 5-point rating scales were developed (see Figure 4-1). These scales were drawn from McCormick's Position Analysis Questionnaire (McCormick, 1979). These were selected because of their proven psychometric qualities and because they are easily understood. 
Table 4-1

Truck Driving Tasks

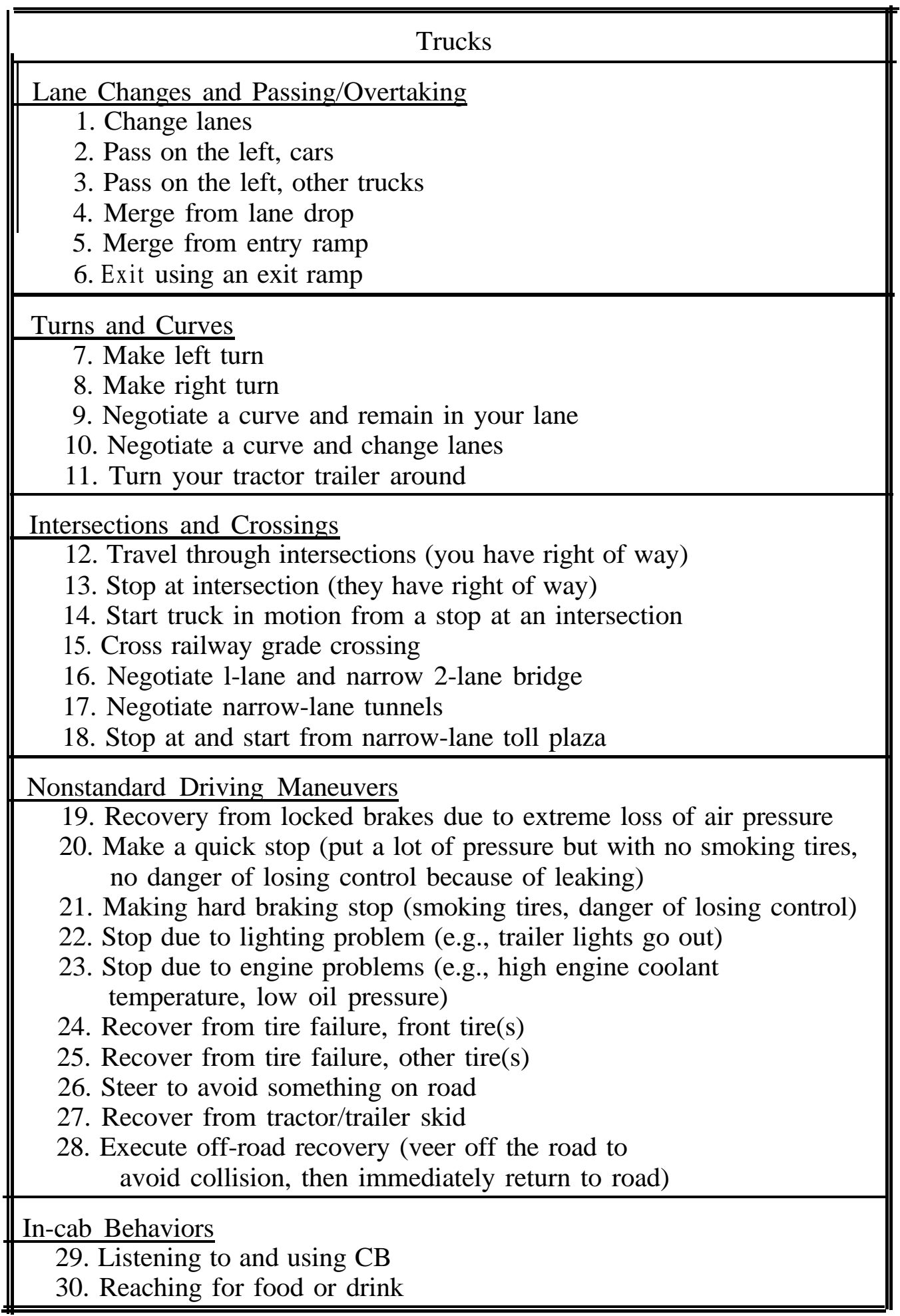


Figure 4-1

Difficulty and Importance to Safety Scales

\section{DIFFICULTY}

$\begin{array}{ccccr}1 & \mathbf{2} & \mathbf{3} & \mathbf{4} & \mathbf{5} \\ \begin{array}{c}\text { Very } \\ \text { Easy }\end{array} & \text { EaSy } & \begin{array}{c}\text { Average } \\ \text { Difficulty }\end{array} & \begin{array}{c}\text { High } \\ \text { Difficulty }\end{array} & \text { Dxtreme } \\ \text { Difficulty }\end{array}$

\section{IMPORTANCE TO SAFETY}

$\begin{array}{lcccc}1 & \mathbf{2} & \mathbf{3} & \mathbf{4} & \mathbf{5} \\ \begin{array}{l}\text { Very } \\ \text { Minor }\end{array} & \text { Low } & \text { Average } & \text { High } & \text { Extreme }\end{array}$


The identification of subjects, i.e., truck drivers, for this study was relatively straight-forward. It was decided not to restrict the sample of drivers in any way. That is any age, any amount of experience, any type of rig driven, etc., was acceptable. This approach provided, within the sample of drivers, a wide range of truck driver experience and background. Although it was desirable to have drivers who have experienced a wide range of conditions and tasks, such subjects were not sought out. However, because the drivers were to be sought out at a truck stop that caters to long haul drivers, it was anticipated that the subjects would most likely have a wide range of experience.

\section{$\underline{\text { Objectives }}$}

The objective of this effort was to collect, from a sample of professional truck drivers, data on the perceived difficulty and safety criticality of selected driving tasks. It was also of interest to determine how the perceived difficulty and safety criticality of selected driving tasks varied across driving conditions which spanned the range of driving demand.

\section{Subjects}

The subjects consisted of 30 volunteer truck drivers interviewed at a large, multi-facility truck stop along a major interstate highway . These drivers drove a variety of rigs and tractor types, ranged in age from 25 to 58 years (average 41.5), and ranged in experience from 1 to 42 years (average 15.7).

\section{$\underline{\text { Procedure }}$}

Each of the two scales and each of the three conditions were printed on 3 by 5 inch laminated card stock. The instructions and data sheets, which were never shown to the drivers, were prepared on regular paper for the data collector.

Each of the 30 tasks was assigned to one of six lists for presentation to the subjects. Each task appeared on only one list; the six lists were mutually exclusive and collectively exhaustive in the tasks they contained.

Each driver was given five tasks which were judged on safety criticality and difficulty in each of the three selected driving conditions. The order of presentation of the tasks and conditions was randomized to control for order effects. Thus, each subject made 15 difficulty and 15 importance judgments across the task-driving conditions combinations. Each of the six tasks lists was presented to five different subjects in order to obtain replications. In total, the 30 subjects made 900 difficulty and importance-to-safety judgments, which constitute the data for analysis.

The 30 drivers were interviewed at a truck stop. First, the driver was approached by the data collector, who explained the study and requested participation, If the driver agreed to participate, the data collector read the instructions to the driver. The data collector then asked a few demographic questions and begin the data collection. The data collector read a condition, a task statement, and showed printed exhibits (the condition and two scales) to the subject. The data collector noted the subject's response. This sequence was repeated until all data were collected for the subject. The data collector spent approximately 20 minutes with each subject. 


\section{$\underline{\text { Results }}$}

Ratings averages, and standard deviations for each task, under each condition, are presented in Table 4-2. Table 4-2 also includes the means and standard deviations for the ratings for each condition across all tasks. There appear to be substantial differences in the means of the difficulty ratings across driving conditions. The same is true for the averages in importance-to-safety rating means across driving conditions.

Note in Table 4-2 that the differences between the rating means between conditions vary in degree. Although the rating means on the difficulty scale and the importance-to-safety scale increase as the driving conditions worsen, the difference between Conditions 1 and 2 is much larger than between Conditions 2 and 3. It appears that Conditions 2 and 3 are much closer in severity of conditions than is indicated in the factor ratings shown in Table 3-2. this suggests that driving task difficulty and safety criticality increase monotonically relative to perceived driving demands, but this is not a linear relationship.

Upon examination of the individual task ratings shown in Table 4-2, the results show:

- $\quad$ Tasks which are most difficult (ranked 4.0 or greater in both conditions 2 and 3) are related to lane changing, recovery from locked brakes, making hard braking stops, recovery from tire failure, recovery from skids and executing off-road recovery.

- $\quad$ Tasks which are most important to safety (ranked 4.0 or greater in both conditions 2 and 3 ) are related to changing lanes, passing cars on the left, merging from entry ramp, making left turn, turning trailer around, starting from stop at intersection, making a railway crossing, negotiating 1-lane or narrow 2-lane bridges, and nearly all nonstandard driving maneuvers.

Even under the best conditions (e.g., lane changes under Condition 1), tasks rated as easy to perform can still be considered average or above in importance to safety. Thus some tasks are not difficult to perform, but when done, must be done correctly or safety can be compromised.

\section{$\underline{\text { Conclusions }}$}

As indicated in Section 3.0, the most important factors that determine driving condition demand are traction, visibility, and traffic density. Relative to the "ideal" driving conditions of Condition 1, task ratings were closer between Condition 2 (where good traction was available but all other factors were set at "poor" levels) and Condition 3 (where traction, visibility, and traffic density were poor but other factors were set at "good" levels). This is consistent with the results of the demand assessment on the relative importance of traction, visibility, and traffic density (See Section 3, Table 3-1). From this we gain additional confidence that if a test scenario is to have a significant level of workload associated with the primary driving task, one or more of these factors must be degraded.

There were commonalities among tasks judged most difficult and most important to safety. These can be described as those tasks that require high visual/perceptual demand and precise or quick steering/braking control. Again, this is consistent with the demand analysis. These task 
Table 4-2

Difficulty and Importance to Safety Ratings of Driving

Tasks Under Three Driving Conditions

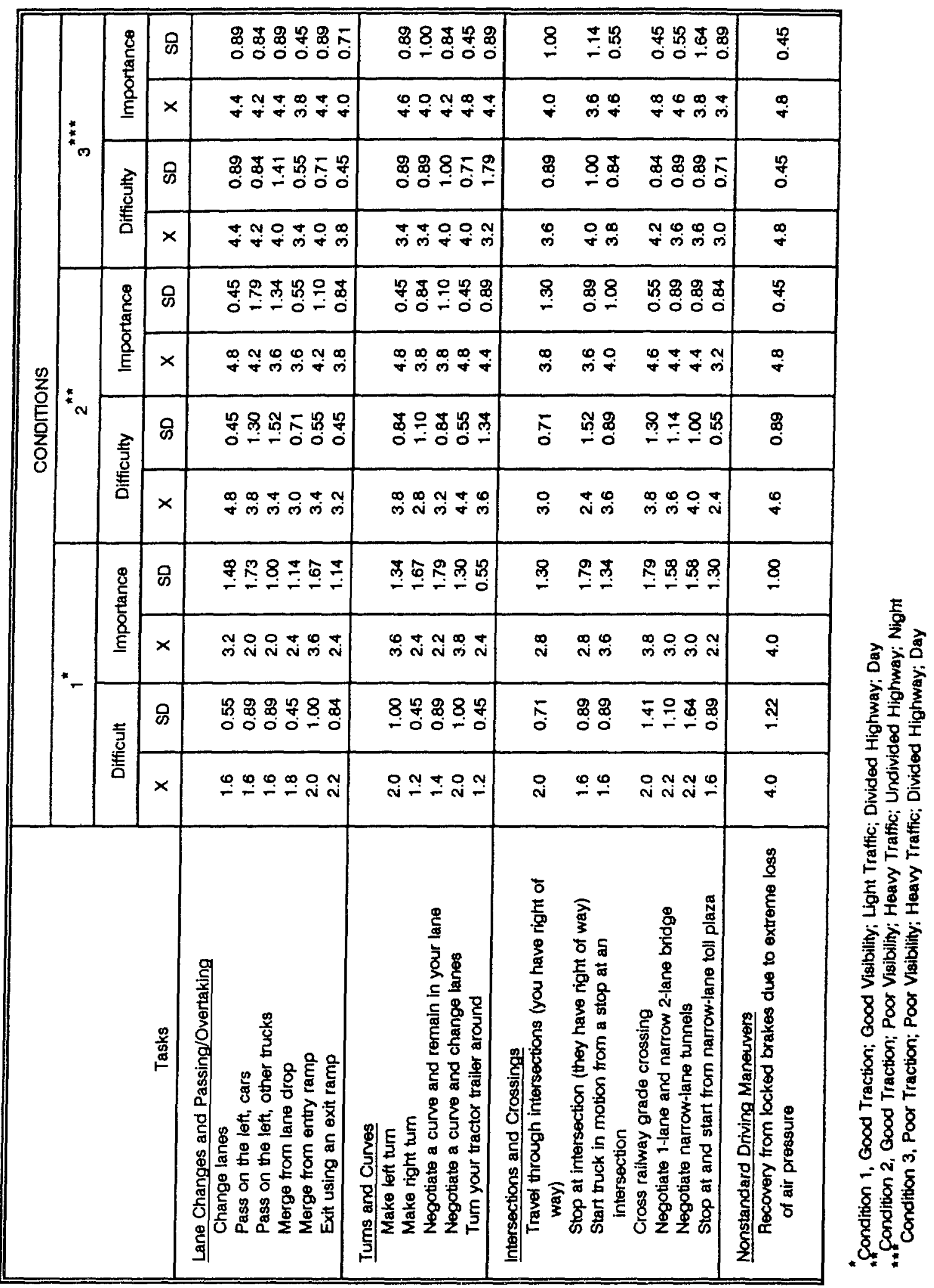


Table 4-2

Difficulty and Importance to Safety Ratings of Driving Tasks Under Three Driving Conditions (Continued)

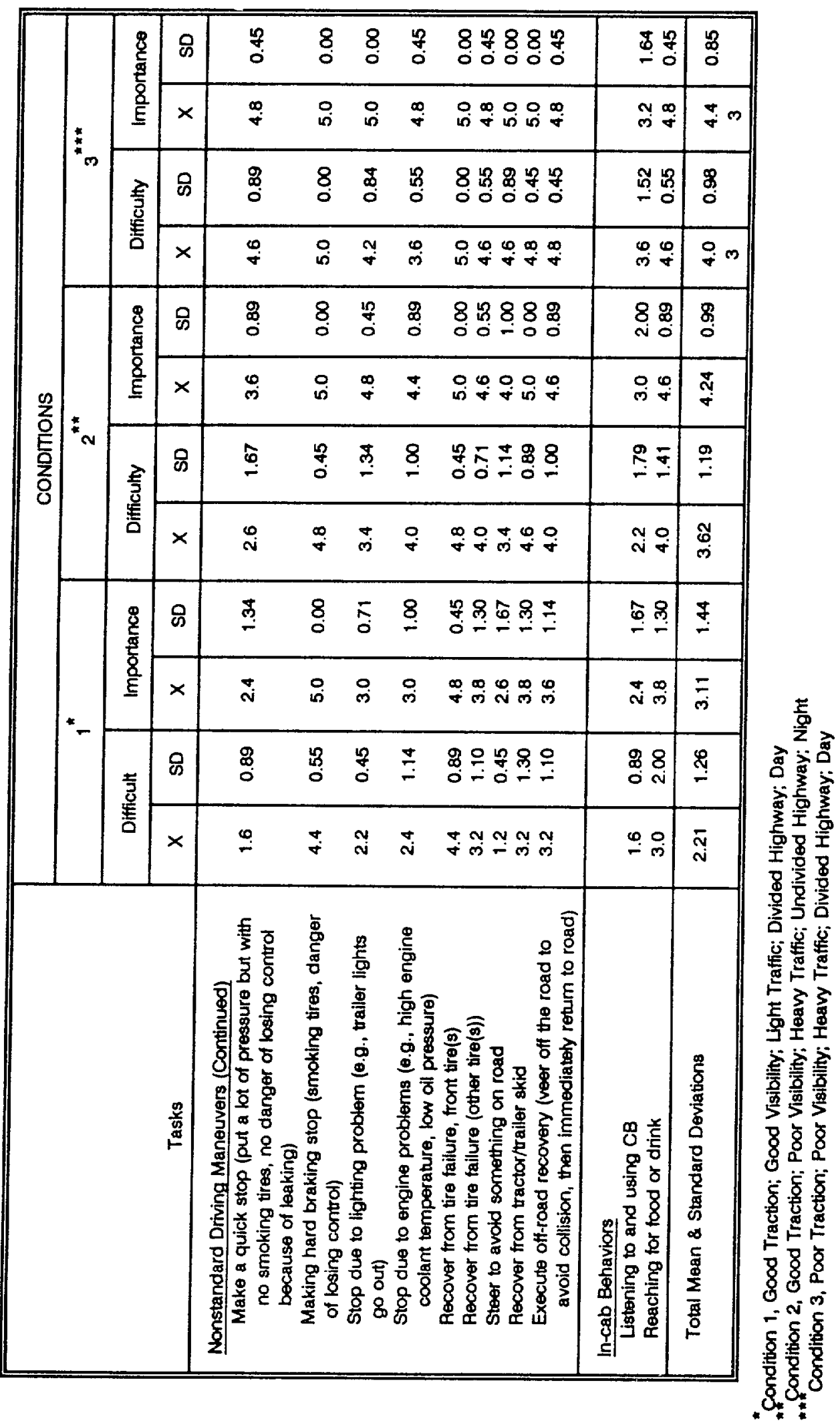


characteristics are precisely those that would be most affected by poor traction (which affects control over vehicle position and path) as well as poor visibility (which affects the driver's ability to visually maintain a thorough situation assessment) and high traffic density (which introduce other vehicles).

From this we conclude that test scenarios should contain opportunities to observe driver performance and behavior during the more important and difficult driving tasks as indicated in this report. 


\section{References}

(1) McCormick, E. J. (1979). Job Analysis: Methods and Applications. New York: AMACOM. 


\title{
5.0 DESIGN OF FIELD OBSERVATIONS OF TRUCK DRIVERS
}

\author{
Introduction
}

One of the primary objectives of Task 3 was to acquire heavy truck driving task analysis data from field observations of drivers to supplement existing data presented in the open literature. The literature on task analyses of driving was reviewed and reported in the Task 1 Interim Report titled "Task Analysis Data and Protocols Review." The data collected in Task 3 provides additional insights into the tasks performed by truck drivers. The new data will assist in the development of workload evaluation protocols later in the project. This section of the Task 3 report describes the design of the field observations used to acquire the supplemental task analysis data.

\section{Objectives}

The specific objectives of field data collection efforts were to conduct on-the-road observations of professional truck drivers to:

Provide preliminary estimates of the visual allocation used by truck drivers during normal driving, and to estimate the frequency and duration of eye glances by location.

- $\quad$ Characterize the allocation of the drivers' manual resources (the hands) to driving tasks. The percent of time the drivers' hands were involved with steering the truck or available for activation of in-cab controls were of particular interest in this effort.

- $\quad$ Estimate the number of glances and glance durations typically required by the drivers to perform selected motor and visual tasks.

- $\quad$ Assess the feasibility of using video recording techniques to acquire visual workload data of truck drivers in an on-the-road study for both day and night operations.

A combination of video recording and work sampling techniques (Smith, 1978) was used to acquire the data.

\section{Video Data Acquisition Equipment}

A video data acquisition system was developed and used to record subject eye glance locations and durations, and the road scene ahead of the truck. Eye glance locations included the left side mirror, right side mirror, instrument panel, and the controls above in the header panel (i.e., the $\mathrm{CB}$ or AM/FM stereo radio).

The video system, depicted in Figure 5-1, consisted of the following items:

a video camera (with infrared light source for night driving) directed at the driver's face a video camera directed at the road scene ahead of the truck video inserter/combiner 
Figure 5-I Diagram of Video Recording System

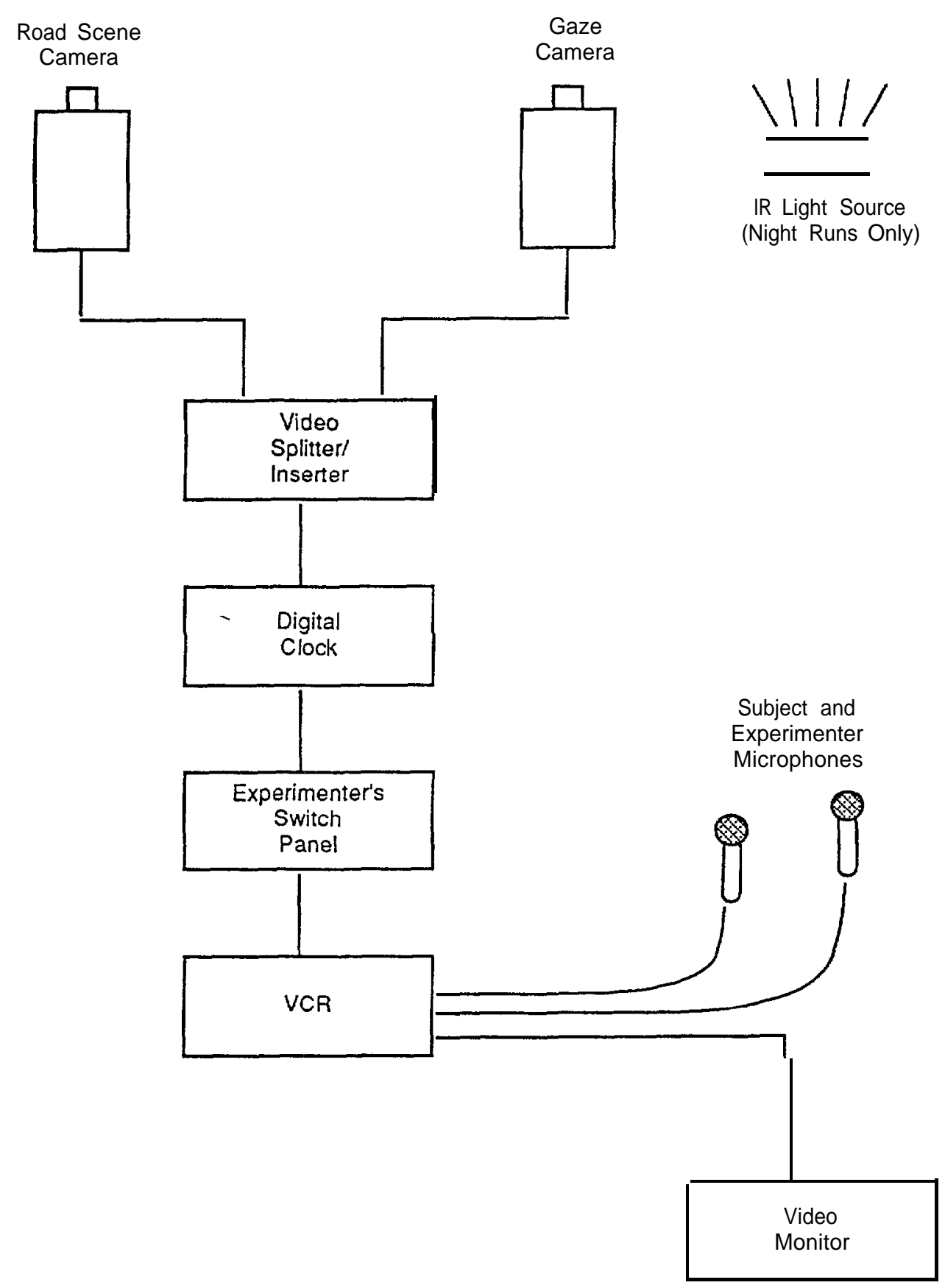




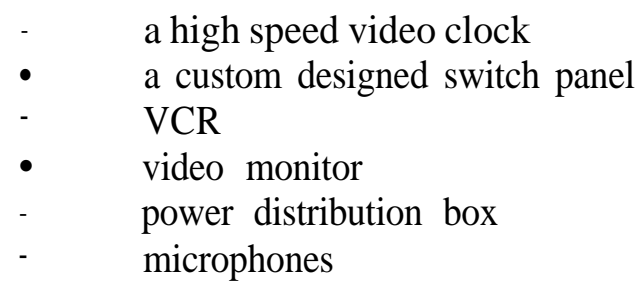

The road scene and face cameras were installed on small, compact tripod heads and mounted to the top of the instrument panel (IP) Since the cameras were mounted using existing bolt holes in the IP, no permanent alterations were made in the tractor to accommodate the equipment. The cameras were positioned in locations that did not interfere with the driver's field of vision. The cameras were also rigidly mounted to the tractor. Preliminary tests conducted by the project staff at NHTSA's Vehicle Research and Test Center indicated no serious effects on picture quality resulted from road vibrations when the cameras were mounted in this fashion. The remaining equipment was positioned on the floor of the cab or on the driver's bed in the sleeper box. Installation and checkout of the video system typically required one hour.

The video acquisition equipment allowed the road scene ahead and the driver's face to appear as a split-screen image on the VHS tape. A custom-built switch panel to allowed the experimenter to dialin codes that represented changing driving conditions. The switch panel consisted of six lo-position thumbwheel switches, which provided a digital input that was superimposed on the video image. In essence, the switch panel served as a video log for the experimenter. It permitted limited amounts of digital information to be coded on the tape with the road scene and driver's glance data.

The digital clock established a time base for each run. The clock was also superimposed on the video image during the recording process and became a permanent part of the tape.

Microphones were placed in the cab to allow the experimenter's commands to be recorded on the tape. Driver commentary was also recorded on the tape.

To facilitate data collection on night runs, a custom-built infrared (IR) light source was used as supplemental lighting on the subject's face. Because the IR light was not detectable by the subject and the camera was sensitive into the IR portion of the electromagnetic spectrum, this technique provided an effective method for illuminating the driver's face at night.

The experimenter checked the recording process by viewing an on-board video monitor and listening to the audio track being recorded during equipment setup and while on-the-road.

All equipment was powered from the truck's 12 VDC electrical system.

\section{$\underline{\text { Subjects }}$}

Nine subjects participated in this study. The subjects were active, professional drivers from a Columbus-based regional long-haul carrier. The subjects volunteered for the study and were paid $\$ 40.00$ for their participation. Each subject made one run in the study. 
Table 5-1 summarizes the subjects' biographical data recorded during the run. As shown in Table 5-1 the subjects ranged in age from 28 to 56 years with a mean of 38 years. Their years of driving experience ranged from 4 to 42 years with a mean of 15.8 years. All of the subjects were male. Five of the nine subjects wore corrective lenses. All subjects were judged to be skilled and experienced drivers.

\section{Trucks Used}

All trucks used in this study consisted of a conventional tractor and a $48 \mathrm{ft}$. van.

The tractors used were those assigned to the driver by the trucking company. The tractors included recent models manufactured by Kenworth, International, Freightliner and Peterbilt. Since the tractors were assigned, no attempts were made to control the tractor type or the specific cab features for this study. However, it should be noted that there were variations across the models in the basic design of the cab interior, the layout of the controls and displays, and number and types of mirrors used. Table 5-1 illustrates this point for the CB and AM/FM stereo radios.

All of the tractors used in the study were equipped with electronic AM/FM cassette radios, although two had radios that were inoperative at the time of the study. All but two of the tractors had electronic citizen's band radios.

\section{Test Runs}

The data collected during the study were obtained under actual operating conditions on the normal runs to which the subjects were assigned. Because the run departure time, destination and, hence, the route were fixed when a driver volunteered to be a subject, it was not feasible to institute controls to balance the data across a variety of road type or traffic conditions. These factors and the environmental factors were simply observed as they occurred during the course of the run taken by the subject.

In total, nine runs were made during May and June, 1992. The runs represent approximately 35 hours of driving across the nine subjects. Due to power problems in the truck's 12 VDC electrical system much of the visual glance data for Subject 5 was not used in the analysis reported in the next chapter.

The test runs were about evenly split between day and night operations. Table 5-2 summarizes the runs and the types of visual data recorded during each. The majority of the data recorded in the study (estimated in excess of 95\%) were collected on rural interstate highways during periods of relatively light traffic. 
Table 5-1

Subject Biographical Data

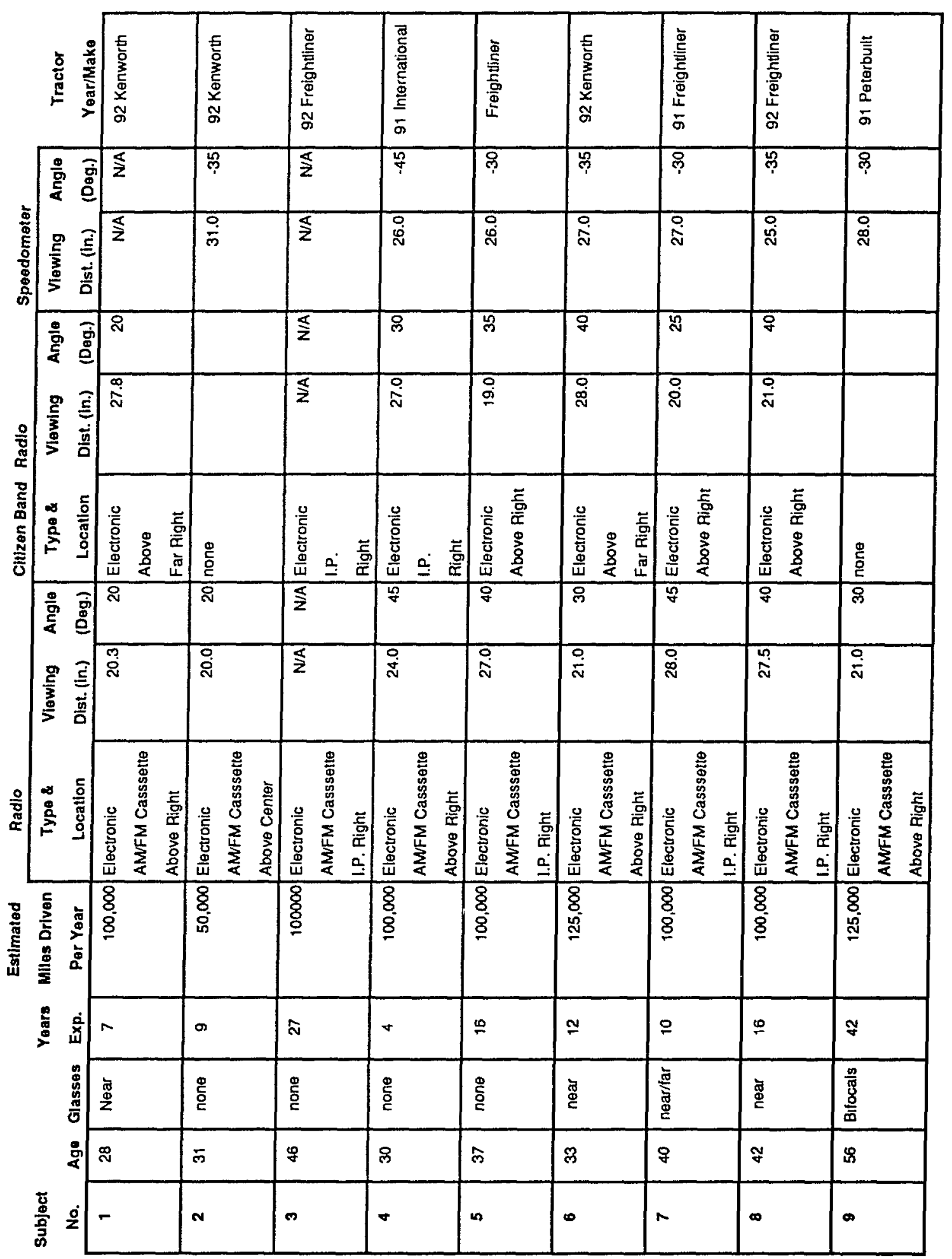

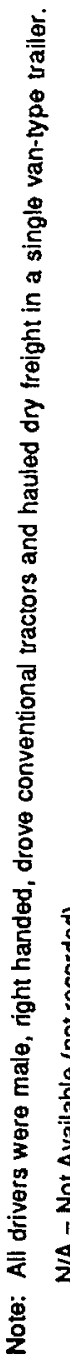


Table 5-2

Summary of Data Collection Runs

\begin{tabular}{|c|c|c|c|c|c|c|c|c|c|}
\hline 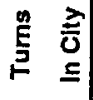 & & $\times \frac{\bar{n}}{z}$ & & & . & & & & \\
\hline 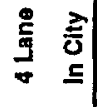 & & $\times \frac{\bar{n}}{2}$ & & & . & & & & \\
\hline $\begin{array}{ll}\stackrel{2}{\vec{E}} & \overline{\overline{0}} \\
\stackrel{\vec{u}}{*} & \underline{E}\end{array}$ & & $\times \frac{\bar{z}}{2}$ & & & . & & & & \\
\hline 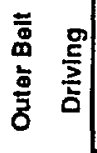 & $\times \quad$ & $\times \frac{-}{z}$ & $\frac{\mathbf{z}}{\mathbf{z}}$ & 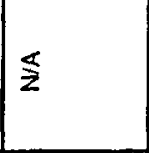 & . & $\times \bar{z}$ & & $\times \bar{z}$ & \\
\hline 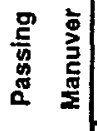 & & & & & . & {$\left[\begin{array}{ll}\infty \\
\times & \text { 11 } \\
& 2 \\
\end{array}\right.$} & $\begin{array}{r}0 \\
\times \quad \begin{array}{r}11 \\
z \\
\end{array} \\
\end{array}$ & & \\
\hline 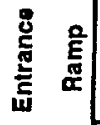 & $\times \frac{n}{n}$ & $\times \begin{array}{l}n \\
\times \quad 2 \\
2\end{array}$ & 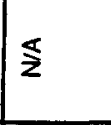 & $\times \quad \frac{n}{2}$ & . & $\times \begin{array}{l}\text { n } \\
\text { n }\end{array}$ & $\times \quad \begin{array}{l}n \\
\times \\
z\end{array}$ & & \\
\hline 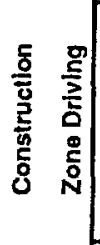 & $\times \frac{-}{2}$ & $\times \frac{\bar{n}}{z}$ & $\times \frac{\bar{n}}{z}$ & $\times \frac{7}{2}$ & . & 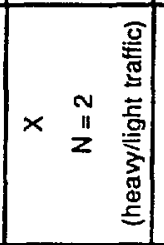 & 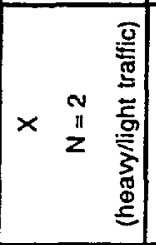 & $\times \frac{-}{2}$ & $\times \frac{\bar{n}}{2}$ \\
\hline 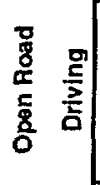 & $\times \quad \overline{2}$ & $\times \quad \frac{\pi}{2}$ & $\times \frac{7}{2}$ & 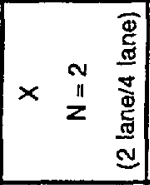 & . & 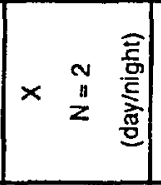 & $\times \frac{-}{11}$ & $x \frac{\bar{z}}{z}$ & $x \frac{\pi}{2}$ \\
\hline 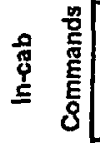 & $\begin{array}{r}N \\
\times \quad 11 \\
2\end{array}$ & $\begin{array}{r}N \\
\times \quad Z \\
z\end{array}$ & $\times \frac{N}{2}$ & $\times \begin{array}{ll} & \\
\times & \text { II } \\
z\end{array}$ & $\times \frac{\bar{y}}{z}$ & $\begin{array}{r}\times \\
\times \quad \\
2\end{array}$ & $\begin{array}{r}0 \\
\times \quad \\
2\end{array}$ & $\times \frac{N}{n}$ & $\times \quad \begin{array}{l}0 \\
x \\
2\end{array}$ \\
\hline 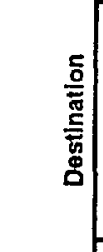 & 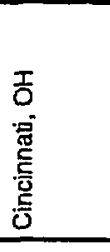 & 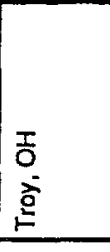 & $\begin{array}{l}I \\
0 \\
\vdots \\
5 \\
\vdots \\
\end{array}$ & 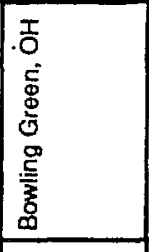 & 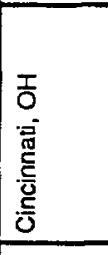 & 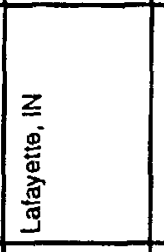 & 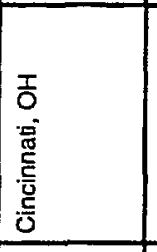 & 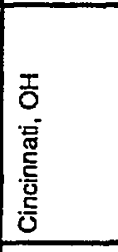 & 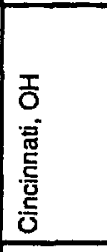 \\
\hline 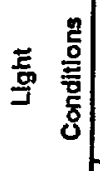 & 命 & 棁 & $\mid$\begin{tabular}{|l}
$\mathbf{r}$ \\
$\frac{\mathbf{n}}{z}$
\end{tabular} & 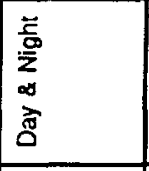 & 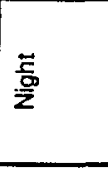 & 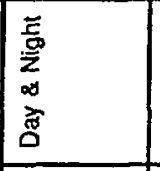 & 商 & \begin{tabular}{|l}
$\bar{E}$ \\
$\mathbf{I}$
\end{tabular} & 空 \\
\hline 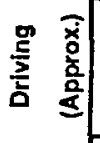 & $\sigma$ & 盀 & m & 0 & $\sigma$ & $\stackrel{n}{+}$ & in & $\infty$ & is \\
\hline 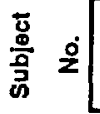 & - & N & n & $\theta$ & $\infty$ & 0 & N & $\infty$ & $\infty$ \\
\hline
\end{tabular}




\section{Data Collection Protocol}

The procedure used for this study was as follows:

A. Pre-Trip Events

1. Install the video equipment in the cab

2. Give instructions to the subject

3. Conduct the pre-trip calibration of subject's head and eye glance location for specified locations

B. Over-the-Road Events (Data Collection)

1. Observe naturalistic driving (day-night, open road, construction zone, etc., as condition were observed)

2. Request the driver execute "commanded tasks" (i.e., directed use of mirrors, radio/CB and instrument panel)

3. Collect data on driving conditions

4. Observe driver's hand activity at selected times

C. Post-Trip Events

1. Debrief the subject at the end of the run.

2. Remove equipment from cab of truck.

Each of these elements in the protocol is briefly discussed in the following sections.

\section{Pre-Trip Events}

A fixed set of instructions was read to the subject by the experimenter following the installation of the video equipment . These are presented in Appendix 5A. The instructions generally described the study and the tasks the subject would be asked to perform. The instructions stressed the importance of safe driving operations. Subjects were specifically told to operate the truck first and respond to the experimenter's requests only when safe to do so. The experimenter then answered any questions the subject had and asked the subject to sign a informed consent form.

The experimenter conducted the calibration of the subject's head and eye glance locations to selected locations in the cab and road scene prior to departure. In this procedure, the subject was asked to look at a specified location while the subject's head and eye positions were recorded on video tape. This record of the subject's head and glance locations served as a point of reference for the data reducer when classifying the glance locations of the subject.

\section{Over-The-Road Events}

During the over-the-road segment of the run, the manual activity and visual glance data were collected. The following discussion generally describes the activities that occurred. Although the 
discussion implies a fixed order of events, in actuality the order of run events varied depending on the conditions encountered and the expected duration of the run. Hence, the experimenter could exercise considerable latitude in conducting the over-the-road portion of the protocol to take maximum advantage of the conditions that were encountered in the run and to allocate time to all data collection activities. For example, the experimenter might suspend requesting the subject to perform tasks to enable the natural glances through a construction zone to be recorded. After passing the construction zone, the experimenter would resume the commanded tasks again.

In general, no data were collected for analysis until about 30 minutes into the run, which provided an opportunity for the subject to acclimate to the situation.

\section{Observe Naturalistic Driving}

The natural glances made by the driver were recorded on video tape along with the road scene ahead of the truck. The subject was not asked to perform any tasks during this phase of the run. Thus, the visual and manual activities observed during this phase were initiated by the driver.

\section{$\underline{\text { Execute Commanded Visual and Motor Tasks }}$}

At appropriate points in the run, the experimenter asked the subject to execute up to 28 visual or manual tasks typically performed by truck drivers. These commands, listed in Section 6.0, Table 6-3, included $\mathrm{AM} / \mathrm{FM}$ and $\mathrm{CB}$ radio operations, IP gauge reading, $\mathrm{AC} /$ heater operations and mirror use. The actual number of commands executed by each subject varied because of equipment differences across tractors and differing run lengths (number of replications permitted). To ensure that the commands were executed under conditions similar to normal driving, the 28 commands were requested over approximately 25 minutes of driving time.

The subject's glance locations and durations for each task executed were recorded on the video tape for subsequent data reduction.

\section{Observe Driver's Hand Activity}

A detailed work sampling of the subject's hand activity was conducted at selected times during the run. Observations were taken randomly at one minute intervals during a sampling period and manually recorded on a data sheet. The hand activities were classified into the categories listed in Table 5-3. The amount of time allocated to observing the subject's hand activities varied from subject to subject, depending on the expected length of the run and the driving conditions encountered. During the study the observation time for hand activity ranged from approximately 30 minutes for Subject 5 to about 2.5 hours for Subject 7.

Open road rural highway driving dominated the conditions under which the hand activity was observed. Limited amounts of city driving and driving in freeway construction zones were also observed. 
Table 53

\section{Left Hand and Right Hand Activity Categories \\ Used In Manual Activity Sampling}

\section{Left Hand}

Steering (Hand gripping rim or spokes of wheel)

At Rest on Wheel (Hand on wheel but no grip used)

At Rest Other Location

Activating Horn

Activating Turn Signal

Operating Window

Activating Other Control

Reaching

Personal Adjustment (e.g., Scratch, Adjust Clothing or Glasses)

Eating, Smoking or Drinking

Miscellaneous Other Activity \{e.g., read wrist watch)

\section{Right Hand}

Steering (Hand gripping rim or spokes of wheel)

At Rest on Wheel (Hand on wheel but no grip used)

At Rest Other Location

Shifting Gears

Using CB or Stereo Radio

Activating Other Control

Reaching

Personal Adjustment (e.g., Scratch, Adjust Clothing or Glasses)

Eating, Smoking or Drinking

Miscellaneous Other Activity (e.g., read wrist watch) 


\section{Collect Data on Driving Conditions}

The experimenter performed a modified work sampling of driving tasks and conditions encountered during the run. Observations were taken at 15 minutes fixed intervals and the information manually recorded on data sheets by the experimenter. Information recorded by the experimenter included road type, number of lanes, posted speed limit, vehicle speed, light condition, precipitation and driving maneuver. These data are reported in Section 6.0.

\section{Post-Trip Activities}

At the end of the run, subjects were debriefed to obtain their subjective views of truck driver workload. They were asked to compare the run with others to determine if anything unusual occurred. They were also asked to comment on the study. Biographical and anthropometric data was also collected at this time. Following the debriefing, the subject was given renumeration and the equipment was removed from the cab of the truck.

\section{Data Reduction}

Video Tape Data

The video tapes were manually examined by a data reducer to obtain the glance locations, glance frequency, and glance durations for selected periods of normal driving and for the commanded tasks. In reducing the data, a glance began when the eyes first started to move away from the road scene and ended when the eyes returned to the road scene or to another location. The superimposed high speed clock time was noted at the beginning and end of the glance to calculate the duration. Resolution to 1/30 second, or a single video frame, was possible using this technique.

For normal driving, the glances were classified into nine categories. These were:
A. Left Mirror
B. Right Mirror
C. Instrument Panel
D. Header (Up)
E. Road Ahead
F. Road - Right
G. Road - Left
H. Right Other
I. Left Other

The Left Mirror and Right Mirror categories referred to glances to the left side and right side West Coast mirrors on the truck. The Header (Up) referred to glances to the panel above the driver's head, over the windshield. In this study, the CB and AM/FM stereo radios were frequently found in this location as was the wiper switch in some tractors. The Road-Right and Road-Left categories refer to glances that were to the road scene but not directly ahead of the truck. Glances to these locations were made by the drivers to monitor traffic in adjacent lanes or targets along the edge of the road. 
For the commanded tasks, glance frequency and duration were obtained from the video tape and tabulated for each command executed by the subject.

\section{Work Sampling Data}

The data from the work sampling of subjects' hand activity were cross-classified by simultaneous left hand and right hand activities, then tabulated across all subjects for analysis. The data from the work sampling of driving conditions were also tabulated to obtain frequency counts and percentages by category of information, The results of both work sampling efforts are reported in the next chapter. 


\section{References}

(1) Smith, G. L., Jr. (1978). Work measurement: a systems approach. Columbus, OH: Grid Publishing. 


\section{Appendix 5A \\ General Instructions for Test Subjects}

Thank you for agreeing to help us in this study. The major objective of the study is to better understand the various driving tasks truck drivers must perform and the effects of different highway, traffic and weather conditions on driving. One of the key questions of the study is where drivers look while performing their tasks.

To record data for the project, we have mounted two video cameras in the cab. One video camera is mounted on the dash and is focused on your face to record your glances. The second camera is directed to the road ahead to record traffic density, road conditions and the weather.

During the run we will observe you as you naturally drive. We will also ask you to perform ordinary driving related tasks throughout the trip. For example, we will ask you to use your mirrors, read the speedometer or other engine gauges and operate your radio. Since we will frequently ask you to perform these tasks during the trip, we hope you will be patient with us.

It is essential for you to understand that safe driving comes first Drive the truck first and perform the other tasks only when it is safe and convenient for your to do so. Never blindly follow our instructions if you feel the safety of the trip would be jeopardized. Always obey all rules of the road.

Once again, remember that this is an ordinary trip with the addition of driving related tasks. Safety comes first; execute the other tasks only when it is safe to do so.

You may stop the study at any time during the trip should you be unable to continue. As a token of our appreciation for your assistance, you will be paid $\$ 40.00$ for the trip. We also want to assure you that your name will remain anonymous - only our immediate project staff will know who you are.

Thanks again for your assistance.

Do you have any questions? 


\title{
6.0 RESULTS OF FIELD OBSERVATIONS OF TRUCK DRIVERS
}

\author{
Natural Glance Allocation During Open Road Driving \\ on Rural Freeways
}

A nominal three minute segment of open road, rural freeway driving was sampled from the video tape for each subject. For four of the subjects, the light conditions were night, as these were essentially night runs. These segments were well into the trip and during a period where no commands were issued to the driver. In the analysis of natural glances, glance durations less than 0.25 seconds were not included, because eye movement literature suggests that drivers require a minimum amount of time to extract meaningful information from the visual scene. For example, it is well known that drivers make three to four fixations per second in highway driving, which results in fixation durations of 0.25 to 0.33 seconds. For this reason, a 0.25 second cutoff was selected. Approximately 2.3 percent of the natural glances reduced in this study were less than 0.25 seconds. These glances were judged to be anonalies resulting from the inability to resolve small differences in eye glance locations using the video recording technique.

For subsequent discussions about the data, emphasis is placed on observations of the descriptive statistics. Inferential statistical tests of "differences" are somewhat premature in light of the inability to have a "controlled experiment" and the confounding effects of subjects and run conditions. The primary purposes of this task was to check out the data collection and reduction system and to establish typical values of visual allocation but not to pursue individual hypotheses.

Table 6-1 represents the descriptive statistics from these segments for each subject and per all subjects combined. The data are classified into the 9 locations (see Section 5.0) but those of greatest importance are the first five: left mirror, right mirror, instrument panel, header, and road ahead. Some subjects did not glance at some locations. For example, during the three minute segments no header glances were noted for four of the eight subjects.

The mean glance duration for the mirrors was 1.33 seconds (s) and $1.47 \mathrm{~s}$ for the left and right mirrors, respectively. These data compare well with similar data reported by Rockwell (1988) and others for passenger car drivers. The instrument panel average of $0.93 \mathrm{~s}$ is similar to that reported in the literature and probably reflects check readings of gauges. The subsection titled "Commanded Visual Tasks" discusses the effect of visual discrimination commands to the drivers.

The value of $0.80 \mathrm{~s}$ for glances to the header reflects the driver looks to the $\mathrm{CB}$ or radio to locate controls. The change in location angle from the road scene to the header is small, less than 45 degrees (vertical). This is similar to the location angle of the speedometer in the instrument panel. There are differences both within and between subjects, as shown later in an example contrasting two night runs of subjects 6 and 8 . Of interest, is the coefficient of variation, the square root of the sample variance divided by the mean. For the header, instrument panel and the two mirrors the coefficient of variation was generally less than $0.5 \mathrm{~s}$ which shows a tight distribution for these glance durations. Since the road scene ahead is essentially the home base from which visual excursions are made to other locations, its glance duration mean and variance are of less significance.

The data can also be examined by the percent of time the driver spends on mirrors, header, instrument panel and the road ahead. Table 6-1 shows that over all subjects, 5.5 percent of the time 
Table 61

Natural Glances by Location

Subject Number (Light Condition During Run)

\begin{tabular}{|c|c|c|c|c|c|c|c|c|c|c|c|}
\hline $\begin{array}{l}\text { Glance } \\
\text { Locatlon }\end{array}$ & & $\begin{array}{r}1 \\
\text { (Day) } \\
\end{array}$ & $\begin{array}{r}2 \\
\text { (Day) } \\
\end{array}$ & $\begin{array}{r}3 \\
\text { (Night) } \\
\end{array}$ & $\begin{array}{r}4 \\
\text { (Night) } \\
\end{array}$ & $\begin{array}{r}6 \\
\text { (Night) } \\
\end{array}$ & $\begin{array}{r}7 \\
\text { (Day) } \\
\end{array}$ & (Night) & $\begin{array}{c}9 \\
(\mathrm{D} a \mathrm{y})\end{array}$ & $\begin{array}{c}\text { All } \\
\text { Subjects Total }\end{array}$ & $\begin{array}{l}\text { \%of } \\
\text { Tlr }\end{array}$ \\
\hline \multirow{6}{*}{$\begin{array}{l}\text { Left } \\
\text { Mirror }\end{array}$} & $\mathrm{N}$ & 10 & 13 & 15 & 4 & 15 & & 3 & 2 & 62 & \\
\hline & Mean & 1.44 & 1.18 & 1.17 & 1.32 & 1.53 & see & 1.13 & 1.63 & 1.33 & \\
\hline & Variance & 0.21 & 0.25 & 0.03 & 0.20 & 0.18 & Note. ' & 0.09 & 0.32 & & \\
\hline & Median & 1.27 & 1.06 & 1.14 & 1.40 & 1.50 & & 1.07 & 1.63 & & \\
\hline & 90th \%tile & 2.12 & 2.03 & 1.34 & 1.77 & 2.13 & & 1.47 & 2.23 & & \\
\hline & TotalTime & 14.40 & 15.34 & 17.55 & 5.20 & 22.95 & & 3.39 & 3.66 & 02.57 & 5.6 \\
\hline \multirow{6}{*}{$\begin{array}{l}\text { Right } \\
\text { Mirror }\end{array}$} & $\mathrm{N}$ & 5 & 4 & 1 & 5 & 5 & 8 & 2 & 2 & 32 & \\
\hline & Mean & 1.79 & 1.65 & 1.66 & 0.86 & 1.79 & 1.33 & 1.37 & 1.42 & 1.47 & \\
\hline & Variance & 0.62 & 0.11 & 0.00 & 0.07 & 1.67 & 0.09 & 0.002 & 0.20 & & \\
\hline & Median & 1.34 & 1.72 & 1.66 & 0.93 & 1.20 & 1.22 & 1.37 & 1.42 & & \\
\hline & 90th \%tile & 2.67 & 1.94 & 186 & 1.13 & 4.23 & 1.73 & 1.40 & 1.73 & & \\
\hline & Total Time & 8.95 & 6.60 & 1.66 & 4.30 & 8.95 & 10.64 & 2.74 & 2.64 & 46.88 & 3.2 \\
\hline \multirow{6}{*}{$\begin{array}{c}\text { IInstrument } \\
\text { Panel }\end{array}$} & $\mathrm{N}$ & 6 & 16 & 11 & 34 & & 3 & 2 & 6 & 80 & \\
\hline & Mean & 1.95 & 0.94 & 1.05 & 0.65 & & 1.10 & 0.73 & 1.19 & 0.93 & \\
\hline & Variance & 0.49 & 0.17 & 0.16 & 0.06 & • & 口ब⿺辶 & 0.002 & 0.13 & & \\
\hline & Median & 1.85 & 1.23 & 0.97 & 0.60 & & 1.13 & 0.73 & 1.10 & & \\
\hline & 90th \%tile & 3.00 & 1.65 & 1.70 & 0.90 & & 1.20 & 0.77 & 1.03 & & \\
\hline & Total Time & 11.70 & 16.92 & 11.55 & 22.10 & & 3.30 & 1.46 & 7.14 & 74.17 & 5. \\
\hline \multirow{6}{*}{$\begin{array}{l}\text { Header } \\
\text { (Up) }\end{array}$} & $\mathrm{N}$ & 3 & 6 & & 4 & 2 & & & & 15 & \\
\hline & Mean & 1.12 & 0.75 & & 0.47 & 1.13 & & & & 0.80 & \\
\hline & Variance & 0.25 & 0.07 & * & 0.004 & 0.14 & & . & . & & \\
\hline & Median & 0.67 & 0.62 & & 0.43 & 1.13 & & & & & \\
\hline & 9ch\%tile & 1.70 & 1.10 & & 0.57 & 1.40 & & & & & \\
\hline & Total Time & 3.36 & 4.50 & & 1.88 & 2.26 & & & & 12.00 & 0. \\
\hline \multirow{6}{*}{$\begin{array}{l}\text { Road } \\
\text { Ahead }\end{array}$} & N & 28 & 54 & 29 & 71 & 38 & 35 & 12 & 25 & 292 & \\
\hline & Mean & 5.08 & 1.93 & 5.09 & 1.77 & 3.23 & 3.81 & 15.36 & 5.67 & & \\
\hline & Variance & 33.59 & 4.41 & 48.04 & 2.65 & 11.06 & 6.66 & 416.34 & 23.00 & & \\
\hline & Median & 2.03 & 1.15 & 2.63 & 1.50 & 2.02 & 3.03 & 4.75 & 4.40 & & \\
\hline & m I \%tile & 15.20 & 4.20 & 16.06 & 3.07 & 6.67 & 7.07 & 40.80 & 12.57 & & \\
\hline & Total Time & 142.24 & 104.22 & 170.61 & 125.67 & 122.74 & 133.35 & 184.56 & 141.75 & 1125.34 & 76.2 \\
\hline
\end{tabular}

NOTE: All data are given in seconds. 
Table 6-I

Natural Glances by Location (Continued)

Subject Number (Light Condition During Run)

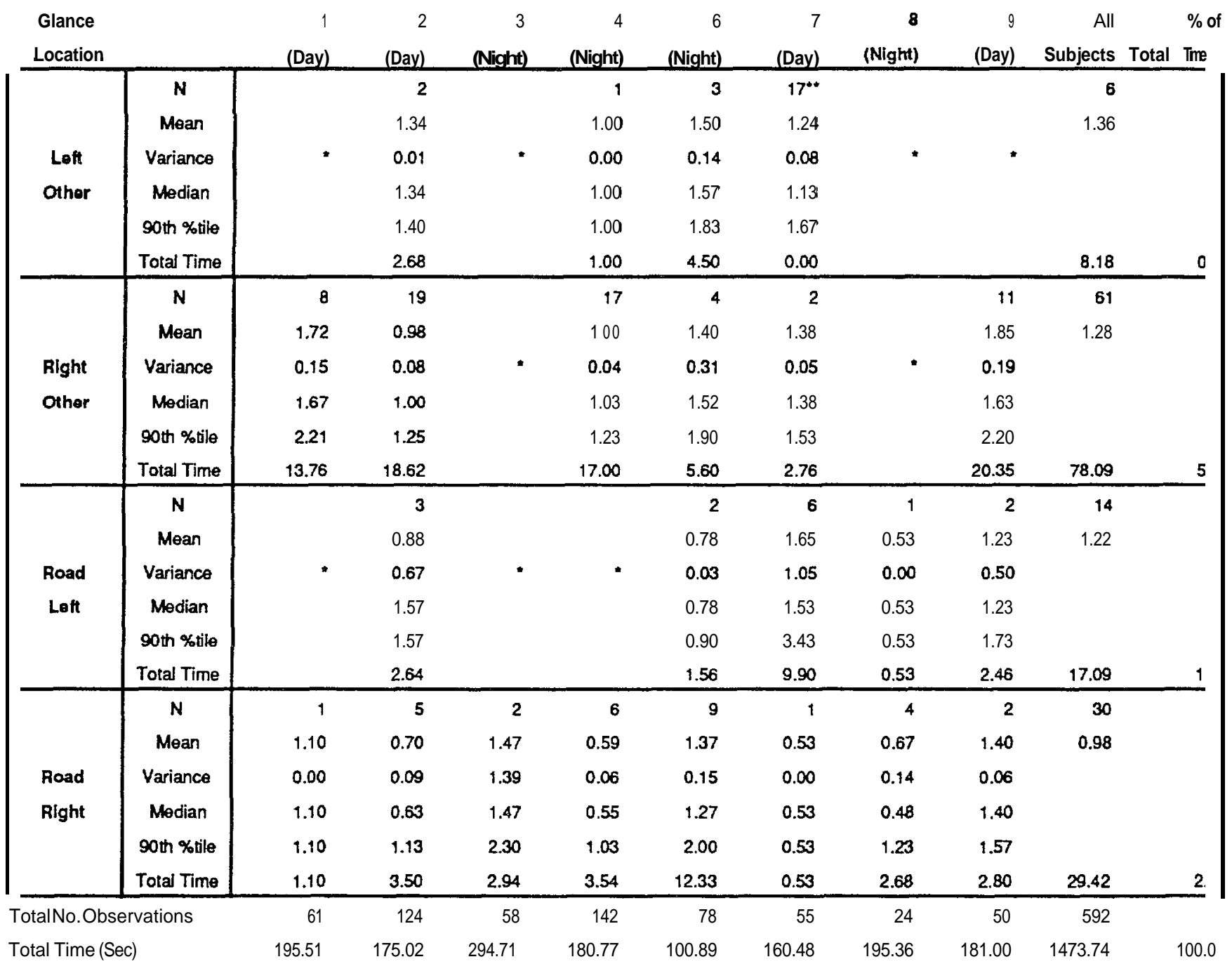

Notes:

All data are given in seconds.

* No glances observed to this location.

- * Left mirror glances could not be resolved for this subject due to unusual looking behavior in that direction. See glances for the "Left Other" location. 
the driver was glancing at the left mirror, 3.1 percent to the right, 5 percent on the instrument panel, 0.8 percent on the header and 75.3 percent on the road scene. The dominance of the left mirror over the right probably reflects the fact that more travel is made on the right lane of travel.

Table 6-2 compares subject 6 with subject 8 both of whom are engaged in night, open road rural driving. Subjects differ widely in their idiosyncratic visual driving strategies. Subject 8 spent 94 percent of the time on the road scene and made few and rapid glances to the mirrors. Subject 6 spends 67 percent of the time on the road ahead and could be characterized as having an active visual search pattern and concerned with positional awareness, for example, the potential presence of overtaking cars.

The presence of an observer may cause a driver to modify his or her scan patterns. For example, a greater than normal emphasis on speed maintenance will promote more frequent speedometer glances. The fair number of "right other" glances probably reflects this type of observer effect.

\section{Commanded Visual Tasks}

As part of the data collection, the experimenter requested that the subject perform a list of commanded visual and motor activities. These were done at periodic intervals over the run. Table 6-3 shows the list of the 28 commands. Since many of these commands were similar (for example, adjust CB volume up or down), the 28 commands were aggregated into 20 categories and organized into common sets (for instance mirror use, instrument reading, radio and $\mathrm{CB}$ operation, and control use).

Visual glance data were recorded during the execution of these tasks, as shown in Table 6-4. This table summarizes the data over all subjects and records both glance fixation and duration as well as the product of glance duration mean and glance frequency mean (namely average time off the road to complete the execution of the task).

The mean glance duration of the mirror data is about 10 percent higher than for the naturalistic runs and probably reflects the drivers added search to provide the "correct" answer. Glances to the left mirror are about 15 percent longer, on average, than comparable glances in an automobile (Rockwell, 1987), though the reliability of this difference is unclear. The mean number of glances to locate traffic in either mirror probably reflects the need to have a confirmation for the first glance. Again, this is probably a "test" effect.

Gauge readings of exact speed require more interpretation and visual discrimination as opposed to check readings which probably accounts for the somewhat longer and more frequent glances to the former than those found in the latter task.

Radio and CB volume adjustments require only enough vision to locate the controls, hence the small glance duration. When it comes to manual tuning, however, an average of over eleven glances of 1.33 seconds were necessary to complete the task, requiring an average of 15 seconds (i.e., mean glance duration times mean glance frequency) off the road scene for execution. For comparable tasks, truck driver average glance duration was uniformly longer than that for cars (Wierwille, Antin, Dingus, and Hulse (1987)). 
Table 6-2

Illustrative Differences in Driver Visual Allocation

$\begin{array}{lrrr}\text { Subject Number } & & 6 & 8 \\ \text { Left Mirror } & \text { Mean } & 1.53 & 1.13 \\ & \text { Variance } & 0.58 & 0.09 \\ & \text { Total Time } & 22.95 & 3.39 \\ & & 12.70 & 1.70 \\ & \text { Percent of Total } & & \\ \text { Right Mirror } & & & \\ & \text { Mean } & 1.79 & 1.37 \\ & \text { Variance } & 1.87 & 0.00 \\ & \text { Total Time } & 8.95 & 2.74 \\ & \text { Percent of Total } & 5.00 & 1.40 \\ & & & \\ \text { Road Ahead } & \text { Mean } & 3.23 & 15.38 \\ & \text { Variance } & 11.06 & 418.34 \\ & \text { Total Time } & 122.00 & 184.00 \\ & \text { Percent of Total } & 67.00 & 94.00\end{array}$

Runs were 3-minute, night, open road, rural freeway natural glances 
Table 6-3

Protocol for Commanded Tasks

\section{Command Name}

1. Radio - Tune Favorite Station

2. Speedometer-Read Exact Speed

3. Read Air Pressure

4. Left Mirror-Detect Vehicle

5. Radio Volume Up

6. Read RPM

7. Change CB Frequency to Ch. 19

8. Adjust CB Squelch

9. CB Volume Up

10. Read Water Temperature

11. Right Mirror-Detect Vehicle

12. Turn Wipers On

13. Turn Wipers Off

14. Read Oil Pressure

15. Read Speed -

\section{Compare w/ Posted Speed}

16. Radio Volume-Down

17. Turn Heat (AC) Up

18. Change CB Frequency to Ch. 3

19. Adjust CB Squelch

20. CB Volume-Down

21. Turn Heat (AC) Down

22. Left Mirror-Discrimination

23. Defrost Fan-Up

24. Defrost Fan-Down

25. Read Fuel Gauge

26. Tune to 103.1 FM

27. Tune Radio to $90.5 \mathrm{FM}$

28. Right Mirror-Discrimination

\section{Instructions to Subject}

Could you tune the radio to your favorite FM station?

What is your current speed?

What is your air pressure reading?

Do you see any traffic in your left mirror?

Could you turn up the volume on the radio?

How many RPMs are you doing?

Can you change the $\mathrm{CB}$ to Channel 19 ?

Now adjust the squelch.

Could you turn the CB volume up a little?

What is your water temperature reading?

Do you see any traffic in the right mirror?

Could you turn on your window wipers?

Okay, you can turn the wipers off.

What is your oil pressure reading?

Are we going above or below the speed limit?

Could you turn the radio volume down?

Could you turn up the heat (AC) a little?

Change the CB to Channel 3.

Now adjust the squelch.

Could you turn the CB volume down?

Could you turn down the heat (AC) a little?

What color is the vehicle to our left rear?

Could you turn up the defroster fan for me?

Okay, you can turn the fan down again.

How much fuel do we have left?

Could you manually tune the radio to 103.1 FM?

Now could you manually tune the radio to 90.5 FM?

What color is the vehicle to our right rear? 
Table 6-4

Summary of Commanded Tasks By Task-All Subjects Combined

Average

Total Average Variance 10th \%tile 90th \%tile Mean Min. Max. Time No. of No. of Glance of Glance Glance Glance No. of No. of No. of Off Command

Trials Glances Duration Duration Duration Duration Glances Glances Glances Road*

\begin{tabular}{|l|cccccccccc|}
\hline Left Mirror - Detect & 21 & 27 & 1.44 & 0.29 & 0.60 & 2.27 & 1.29 & 1 & 2 & 1.85 \\
Right Miner - Detect & 18 & 29 & 1.62 & 0.24 & 0.90 & 2.25 & 1.61 & 1 & 3 & 2.62 \\
Left Mirror - Discrimination & 19 & 30 & 1.77 & 0.34 & 1.07 & 2.57 & 1.58 & 1 & 32.79 \\
Right Mirror - Discrimination & 18 & 38 & 1.58 & 0.18 & 1.07 & 2.03 & 206 & 1 & 53.24 \\
\hline Read Exact Speed & 20 & 29 & 1.50 & 0.35 & 0.77 & 2.43 & 1.45 & 1 & 32.18 \\
Readspeed \& compare & 20 & 27 & 1.40 & 0.33 & 0.67 & 2.30 & 1.35 & 1 & 3 & 1.89 \\
$\quad$ to Posted Limit & & & & & & & & & & \\
Read Air Pressure & 10 & 16 & 1.70 & 0.28 & 0.93 & 2.43 & 160 & 1 & 32.71 \\
Read Engine RPM & 20 & 33 & 1.69 & 0.44 & 1.03 & 2.43 & 1.65 & 1 & 52.79 \\
Head Water Temperature & 7 & 14 & 1.63 & 0.25 & 1.07 & 2.37 & 2.00 & 1 & 3 & 3.26 \\
Read Oil Pressure & 16 & 32 & 1.82 & 0.44 & 0.99 & 2.76 & 2.00 & 1 & 4 & 364 \\
Read Fuel Gauge & 20 & 33 & 1.73 & 0.77 & 1.00 & 2.37 & 1.65 & 1 & 42.86 \\
\hline Radii Volume Up/Down & 32 & 39 & 1.05 & 0.28 & 0.63 & 1.52 & 139 & 1 & 3 & 1.46 \\
Tune Radio to Favorite Sta & 8 & 27 & 1.40 & 0.21 & 0.84 & 1.87 & 3.38 & 1 & 94.72 \\
Tune Radio to Specific Ste & 32 & 310 & 1.33 & 0.24 & 0.83 & 1.90 & 11.31 & 6 & 1815.10 \\
\hline CB Volume Up/Down & 30 & 49 & 0.99 & 0.21 & 0.63 & 152 & 2.00 & 1 & 7 & 199 \\
Adjust CB Squelch & 10 & 20 & 1.07 & 0.14 & 0.62 & 1.65 & 2.60 & 1 & 42.77 \\
Change CB Channel & 30 & 95 & 1.18 & 0.22 & 0.53 & 1.87 & 3.93 & 2 & 54.63 \\
\hline Wipers On/Off & 25 & 27 & 1.00 & 0.12 & 067 & 1.50 & 1.13 & 1 & 21.13 \\
AC Temp Up/Down & 10 & 19 & 1.21 & 0.10 & 0.77 & 1.59 & 2.40 & 1 & 32.89 \\
Fan Speed Higher/Lower & 11 & 17 & 1.43 & 0.13 & 0.93 & 1.93 & 1.71 & 1 & 3 & 245 \\
\hline
\end{tabular}

- Product of the Average Glance Duration (AGD) and Mean Number of Glances (MNG). 
These data match well with earlier research of $\mathrm{R} \& \mathrm{R}$ Research on manual tuning of radios in passenger cars. This suggests that commanded tasks prompt visual allocations which are not substantially different than visual allocations are self-initiated. In effect, when complex visual discrimination tasks are required, drivers use more glances separated by looks to the road rather than increasing the glance duration. The 90th percentile for the commanded tasks shows that, despite the differences in the types and complexity of visual targets, glance durations are usually below 2.5 seconds. This is an important visual allocation strategy by drivers upon which the design of IVHS in-cab system should be based.

Control size probably dictates the number of glances needed to locate the control. Windshield wipers can be manually located with little visual assistance whereas electronic radio volume controls often requires more than one glance. It may be that the larger size of truck cabs require more time to visually acquire in-cab controls and displays. In any event, these data verify the sensitivity of visual allocation measures to different task loads, provides baseline data for future comparisons, and suggests potentially significant differences between truck drivers and car drivers.

\section{Effects of Light Conditions and Traffic Conditions on Natural Glances}

Because most of the runs were either made entirely during daylight or nighttime, any comparison of day vs. night lighting conditions must be confounded by subject effects. Still, since there were four day and four night subjects, we can make a few observations about lighting effect (see Table 6-1). There appears to be little difference in glance allocation (percent of time) for day vs. night for either mirrors, instrument panel, header and road ahead. Average glance duration to the instrument panel appears smaller at night; otherwise, lighting appears to have little effect which could not be explained by subject differences.

Traffic conditions can be examined by looking at Subjects 1, 2, 6 and 8 whose runs occurred under three conditions: open road, rural freeway, outer belt driving, and construction zones. Examination of the outer belt video tapes reveals the presence of more traffic than rural freeways but none of which could be construed as car following. Table 6-5 shows a slight increase in mirror usage and a corresponding reduction in glance duration in outer belt driving. In addition, instrument panel and header glances are fewer in outer belt driving.

Construction zones show greater differences from rural freeway or outer belt driving. Here we note substantially less left mirror use (2.6 percent vs. 7.5 percent for rural freeways), right mirror use (1.7 percent vs. 3.7 percent), and instrument panel samples ( 2.1 percent vs. 4 percent). Correspondingly there was an increase in road ahead glance time (86 percent vs. 74.2 percent for rural freeways).

These data suggest that drivers in construction zones are less concerned with traffic behind them (mirror use) and more concerned with negotiating the construction zone. Again, visual allocation shows sensitivity to changes in driving conditions and suggests an additional element (i.e., construction zones) for inclusion in the to-be-developed protocol.

\section{Manual Activity Sampling}

At fixed intervals during a 2 hour stretch in the runs, the experimenter noted the use of the right and left hands. Table 6-6 shows the categories used to describe the use of either hand for subject 9 . Note that 
Table 6-5

Natural Glances for Subjects 1, 2, 6 and 8 Combined Nominal Three Minute Segment of Driving Time By Location

\begin{tabular}{|c|c|c|c|c|}
\hline $\begin{array}{l}\text { Glance } \\
\text { Locatlon }\end{array}$ & & $\begin{array}{c}\text { Rural Highway } \\
\text { Open Road }\end{array}$ & $\begin{array}{c}\text { Outer Belt } \\
\text { Drfving }\end{array}$ & $\begin{array}{c}\text { Construction } \\
\text { Zones }\end{array}$ \\
\hline $\begin{array}{l}\text { Left } \\
\text { Mirror }\end{array}$ & $\begin{array}{c}\mathrm{N} \\
\text { Mean } \\
\text { MedianoftheVariance: } \\
\text { Time Off Road } \\
\text { \% of Total Time }\end{array}$ & $\begin{array}{r}41 \\
1.37 \\
0.20 \\
56.08 \\
7.5 \%\end{array}$ & $\begin{array}{r}58 \\
1.17 \\
0.07 \\
67.58 \\
9.1 \%\end{array}$ & $\begin{array}{r}17 \\
1.12 \\
0.05 \\
19.07 \\
2.6 \%\end{array}$ \\
\hline $\begin{array}{l}\text { Righl } \\
\text { Mirror }\end{array}$ & $\begin{array}{c}\mathrm{N} \\
\text { Mean } \\
\text { Medianof the Variance } \\
\text { Time Off Road } \\
\% \text { of Total Time }\end{array}$ & $\begin{array}{r}16 \\
1.70 \\
0.47 \\
27.24 \\
3.7 \%\end{array}$ & $\begin{array}{r}25 \\
1.28 \\
0.11 \\
32.10 \\
4.3 \%\end{array}$ & $\begin{array}{r}14 \\
0.68 \\
0.02 \\
12.29 \\
1.7 \%\end{array}$ \\
\hline $\begin{array}{c}\text { Instrument } \\
\text { Panel }\end{array}$ & $\begin{array}{c}\mathrm{N} \\
\text { Mean } \\
\text { Medianofthe Variance } \\
\text { Time Off Road } \\
\% \text { of Total Time } \\
\end{array}$ & $\begin{array}{r}26 \\
1.16 \\
0.17 \\
30.08 \\
4.0 \%\end{array}$ & $\begin{array}{r}16 \\
0.95 \\
0.02 \\
15.18 \\
2.1 \%\end{array}$ & $\begin{array}{r}22 \\
0.72 \\
0.75 \\
15.90 \\
2.1 \% \\
\end{array}$ \\
\hline $\begin{array}{l}\text { Header } \\
\text { (Up) }\end{array}$ & \begin{tabular}{|c|}
$\mathrm{N}$ \\
Mean \\
Median ofthe Variance \\
Time Off Road \\
\% of Total Tirme
\end{tabular} & $\begin{array}{r}11 \\
0.92 \\
0.14 \\
10.12 \\
1.4 \%\end{array}$ & $\begin{array}{r}6 \\
0.99 \\
0.03 \\
5.92 \\
0.8 \% \\
\end{array}$ & $\begin{array}{r}16 \\
0.96 \\
0.10 \\
15.31 \\
2.1 \%\end{array}$ \\
\hline $\begin{array}{l}\text { Road } \\
\text { Ahead }\end{array}$ & \begin{tabular}{|c}
$\mathrm{N}$ \\
Mean \\
Medianofthe Variance \\
Time Off Road \\
$\%$ of Total Time
\end{tabular} & $\begin{array}{r}132 \\
4.20 \\
22.33 \\
553.76 \\
74.2 \%\end{array}$ & $\begin{array}{r}150 \\
3.76 \\
19.35 \\
563.47 \\
76.2 \% \\
\end{array}$ & $\begin{array}{r}107 \\
5.99 \\
71.96 \\
640.96 \\
86.0 \%\end{array}$ \\
\hline
\end{tabular}


Table 6-6

Illustrative Manual Activity Data Subject 9 Rural Highway Driving

Right Hand Activities

\begin{tabular}{|c|c|c|c|c|c|c|}
\hline Left Hand Activitjes & Steering & $\begin{array}{r}\text { At Rest } \\
\text { On Wheel }\end{array}$ & $\begin{array}{r}\text { At Rest } \\
\text { Other }\end{array}$ & $\begin{array}{r}\text { Shifting } \\
\text { Gears }\end{array}$ & $\begin{array}{r}\text { Personal } \\
\text { Adjustment }\end{array}$ & Total \\
\hline Stoering & $\begin{array}{r}53 \\
39.0 \% \\
\end{array}$ & $\begin{array}{r}7 \\
5.1 \% \\
\end{array}$ & $\begin{array}{r}24 \\
17.6 \% \\
\end{array}$ & $\begin{array}{r}1 \\
0.7 \% \\
\end{array}$ & $\begin{array}{r}2 \\
1.5 \% \\
\end{array}$ & $\begin{array}{r}87 \\
64.0 \% \\
\end{array}$ \\
\hline At Rest Wheol & $\begin{array}{r}35 \\
25.7 \% \\
\end{array}$ & $\begin{array}{r}5 \\
3.7 \% \\
\end{array}$ & $\begin{array}{r}2 \\
1.5 \% \\
\end{array}$ & & & $\begin{array}{r}42 \\
30.9 \% \\
\end{array}$ \\
\hline At Rest Other & $\begin{array}{r}1 \\
0.7 \%\end{array}$ & & & & & $\begin{array}{r}1 \\
0.7 \%\end{array}$ \\
\hline Turn Signal & $\begin{array}{r}3 \\
2.2 \% \\
\end{array}$ & & & & & $\begin{array}{r}3 \\
2.2 \% \\
\end{array}$ \\
\hline Operate Window & $\begin{array}{r}1 \\
0.7 \% \\
\end{array}$ & & & & & $0.7 \%$ \\
\hline Personal Adjustment & $\begin{array}{r}1 \\
0.7 \%\end{array}$ & & & & & $\begin{array}{r}1 \\
0.7 \% \\
\end{array}$ \\
\hline Other & $\begin{array}{r}1 \\
0.7 \% \\
\end{array}$ & & & & & $\begin{array}{r}1 \\
0.7 \% \\
\end{array}$ \\
\hline Total & $\begin{array}{r}95 \\
69.9 \% \\
\end{array}$ & $\begin{array}{r}12 \\
8.8 \% \\
\end{array}$ & $\begin{array}{r}26 \\
19.1 \% \\
\end{array}$ & $\begin{array}{r}1 \\
0.7 \% \\
\end{array}$ & $\begin{array}{r}2 \\
15 \% \\
\end{array}$ & $\begin{array}{r}136 \\
\mathbf{1 0 0 .} \mathbf{0 \%} \\
\end{array}$ \\
\hline
\end{tabular}

Notes:

Cell entries are the number of observations and the percent of that cell to the total number of observations.

Data based onapproximatelytwo hours of driving. 
39 percent of the time he used both hands on the steering wheel. The left hand was in a steering position 64 percent of the time compared to 69.9 percent for the right hand.

Table 6-7 combines all 8 subjects in describing manual activity. Both hands were in a steering position on the wheel for an average of 49 percent of the time. Although the variability for this statistic is high, it can be noted that the right hand has spent more time off the wheel (30.1 percent) than the left hand (8.5 percent). This finding is not surprising since the location of most controls, for example, wipers, $\mathrm{CB}$, radio, and heating and air conditioning, are to the right of the steering column. It suggests that drivers allocate manual resources to in-cab tasks other than steering with some frequency. In turn, manual loads imposed by in-cab devices may thereby be a relatively less sensitive measure of workload, unless it is concurrent with visual load.

\section{A Sample Characterization of the Driving Conditions in the Data Collection Runs}

At fixed intervals over the data collection runs, samples of visual glances were collected to characterize the nature of the run driving conditions. Because of the busy schedule of the experimenter, only samples of four to eight observations per run were possible over the run period (usually about three driving hours). Hence, the sample may not reliably characterize the run conditions. Samples included actual speeds, posted speeds, number of lanes, type of highway, traffic density, weather, light conditions, passing or being passed, and whether the truck was in a car following mode. Most of these observations were easily and accurately noted. The noteworthy exceptions were traffic density based subjectively on the number of vehicles in the road scene ahead in the direction of travel and car following based on the subjective estimate of whether a lead vehicle in the lane ahead dictated the driver's speed. The run condition tabulations are found in Appendix 6A.

There was no reason to assume that the participating trucking firm is typical of most trucking operations. This carrier is a regional, long-haul carrier with 6 to 9-hour round trips to such destinations as Toledo, Akron, Cincinnati and Louisville. Rural freeways dominate these runs.

The data collection runs can be characterized as follows:

Over half of the samples were associated with speed limits of $65 \mathrm{mph}$; the remainder were between $45 \mathrm{mph}$ and $55 \mathrm{mph}$, The drivers usually drove at 55 and $65 \mathrm{mph}$. Sixty-one percent of the time the runs were day runs and 92 percent involved dry weather conditions.

- Roadways were typically rural freeways with two to three lanes in the traveled direction,

e Construction zones were encountered on 21 percent of the 77 samples.

- $\quad$ Traffic was found to be light to medium; 77 percent of the time the truck was not overtaken by other vehicles and only 7 percent of the time was the truck passing other vehicles.

Possible car or truck following was observed in 37 percent of the 78 observations.

The last statistic probably reflects momentary car following as caused, for example, by a passing vehicle pulling in front of the driver. In data reduction of the video tapes, the pre-calibrated size of the vehicle ahead on the T.V. monitor was used to establish whether the lead vehicle was within 300 feet of the tractor. In general, it was difficult to find much car following, even when travelling on city outer belts. 
Table 6-7

Percent of Time Engaged in Hand Activities All Subjects Combined Rural Highway Driving

Left Hand Activities

\begin{tabular}{|c|c|r|r|r|}
\cline { 3 - 5 } & & At Rest & All Othor \\
OH Whool
\end{tabular}

Notes:

- Number of subjects with hand activity observed in specified category. Not all subjects produced hand activity in every category.

Total N - 750.

Percentages do notadd to $100 \%$ because of the use of averages for several subjects. 
Thus, it can be noted that most of the time the drivers selected low traffic conditions, minimized workload by avoiding car following and maintained speeds at or near the speed limit on multi-lane rural freeways. All of these behaviors can be considered strategies for management of driver workload.

The only unusual statistic was the percentage of times in conditions of road construction (21\%). It is typical in Ohio to find road construction on rural freeways during the summer months.

\section{Subject Debriefing}

At the end of each data collection session, the drivers were asked a few general questions. Eight of the nine drivers reported that the test run was typical of most runs. One reported excessive unloading by the driver at a load drop off. All agreed the truck handled as usual with no difficulties.

The drivers were then asked to think of driving situations of high workload where workload involved increased visual and manual activity and increased concentration. Responses to this question mirrored the same questions asked of the 34 drivers at truck stops (see Section 2). Traffic, loads, mountain driving and construction zones were most frequently mentioned.

Driver strategies for compensating for these high workload situations again mirrored the truck stop interviews. For example, speed reduction and paying more attention to driving were two general strategies frequently cited. For construction zone traffic tie-ups, one driver suggested he would "pull over and wait."

Three of the nine drivers reported winter driving as the general driving condition involving the most workload.

Finally, when asked about the potential disruption in their driving by the presence of the experimenter, none expressed any concern. Detailed responses are tabulated in Appendix 6B.

\section{Conclusions}

The initial data collection on truck drivers served as a valuable experience on which to design later baseline studies and offer valuable insights on how truck drivers allocate visual and manual capabilities. Some of the general conclusions drawn from these experiences include:

1. Finding a cooperating carrier and cooperating drivers is not an easy, logistical task. Some carrier managers agreed to cooperate but their dispatchers were not motivated to find specific runs (departure times and destinations). When managers and dispatchers would cooperate drivers might fail to cooperate.

2. Departure times often induced hardship on the experimenter, for example, 2:00 a.m. departures.

3. Considerable expense was involved in bringing back experimenters from remote locations for those runs without quick turnarounds to Columbus, Ohio. Bus, motel and meal expenses were often required. In effect, from an experimental testing standpoint, this type of data collection was not efficient. 
4. The ability to achieve different run conditions or to balance run conditions was virtually impossible. In addition, the ability to record data under high density car following traffic was not possible. Drivers would depart at times to avoid traffic, change routes if traffic was suspected to be ahead, or would fall back from traffic ahead to avoid car following.

The above findings point to the wisdom of having a dedicated tractor-trailer and recruiting drivers to operate under specific run conditions for the baseline studies. A dedicated unit also allows special sensors for speed, pedal control positions, etc. to add to the experimental data.

From the data collected there were encouraging results:

1. The two camera system worked very well with only one aborted run due to power problems (Subject 5). The video data for the night runs were remarkably clear. The increased pupil size made data reduction easier. The split screen and high speed clock also made data reduction relatively easy.

2. The glance duration data generally supports the literature in terms of glances to mirrors and instrument panels.

3. Drivers make short excursions from the road scene to get mirror information or to read gauges. Table 6-8 aggregates natural glances for the rural driving conditions by "on road" vs. "off road" (mirrors, etc.).

Here, we note large subject variations in percent of time off the road (3.91\% for Subject 8 to $38.20 \%$ for Subject 2) but smaller differences in mean glance durations. The 90th percentile of such off road glance durations ranges from 1.23 to 2.47 seconds. Despite the confounding of subject and light condition differences, this type of data should be useful in the evaluation of in-cab WI-IS designs. (The 90th percentile of the data reported might argue for a glance duration limit of about 2.25 seconds.) What this means to IVHS design is that, whatever its visual complexity, it must not create glance durations of greater than 2.25 seconds. IVHS display designs must allow accumulation of information serially abetted by successive visual samples. 
Table 6-8

Allocation of Natural Glances By Road and Off Road Locations Rural Highway-Nominal Three Minute Segments of Open Road Driving

\author{
SubjectNumber \\ (Light Condition During Segment)
}

\begin{tabular}{|c|c|c|c|c|c|c|c|c|c|c|}
\hline $\begin{array}{l}\text { Glance } \\
\text { Locatlor } \\
\end{array}$ & & $\begin{array}{c}1 \\
\text { (Day) } \\
\end{array}$ & $\begin{array}{c}2 \\
\text { (Day) } \\
\end{array}$ & $\begin{array}{r}3 \\
\text { (Night) } \\
\end{array}$ & $\begin{array}{r}4 \\
\text { (Night) } \\
\end{array}$ & $\begin{array}{r}6 \\
\text { (Night) } \\
\end{array}$ & $\begin{array}{r}7 \\
\text { (Day) } \\
\end{array}$ & $\begin{array}{r}8 \\
\text { (Night) } \quad \text { ( } \\
\end{array}$ & $\begin{array}{r}9 \\
\text { (Day) } \\
\end{array}$ & Subjects \\
\hline \multirow{8}{*}{$\begin{array}{c}\text { On } \\
\text { Road }\end{array}$} & $\mathrm{N}$ & 27 & 50 & 27 & 65 & 28 & 30 & 7 & 22 & 256 \\
\hline & Mean & 5.31 & 2.22 & 6.44 & 1.99 & 4.89 & 4.8 & 28.81 & 6.68 & 4.28 \\
\hline & Variance & 35.52 & 6.33 & 49.42 & 2.89 & 16.89 & 15.81 & 607.5 & 538.71 & \\
\hline & Median & 2.00 & 1.20 & 3.00 & 1.57 & 3.98 & 3.97 & 26.63 & 4.87 & \\
\hline & 90th \%tile & 16.30 & 4.94 & 16.63 & 3.73 & 11.38 & 10.42 & 65.4 & 18.83 & \\
\hline & Maximum & 26.27 & 13.10 & 24.70 & 9.80 & 14.47 & 17.20 & 65.40 & 21.83 & \\
\hline & Time On Road & 143.37 & 111.00 & 173.88 & 129.35 & 136.92 & 144.00 & 187.671 & 146.96 & 1173.15 \\
\hline & $\%$ of Total Time & 73.36 & 61.80 & 84.90 & 71.40 & 75.58 & 79.21 & 96.09 & 60.36 & $78 . C 91$ \\
\hline \multirow{8}{*}{$\begin{array}{l}\text { Off } \\
\text { Road }\end{array}$} & $\mathrm{N}$ & 32 & 61 & 27 & 65 & 28 & 30 & 7 & 21 & $27^{\prime} 1$ \\
\hline & Mean & 1.63 & 1.12 & 1.15 & 0.80 & 1.58 & 1.26 & 1.09 & 1.71 & 1.211 \\
\hline & Variance & 0.36 & 0.17 & 0.10 & 0.10 & 0.47 & 0.07 & 0.10 & 0.53 & \\
\hline & Median & 1.50 & 1.10 & 1.10 & 0.73 & 1.45 & 1.17 & 1.07 & 1.53 & \\
\hline & 90th \%tile & 2.47 & 1.77 & 1.70 & 1.23 & 2.15 & 1.68 & 1.47 & 2.23 & \\
\hline & Maximum & 3.00 & 2.07 & 1.86 & 1.77 & 4.23 & 1.77 & 1.47 & 4.10 & \\
\hline & Time Off Road & 52.16 & 68.32 & 31.05 & 52.00 & 44.24 & 37.80 & 7.63 & 35.91 & 329.11 \\
\hline & $\%$ of Total Time & 26.70 & 38.20 & 15.10 & 28.60 & 24.42 & 20.79 & 3.91 & 19.64 & 21.911 \\
\hline \multicolumn{2}{|c|}{ Total No. of Glances } & 59 & 111 & 54 & 130 & 56 & 60 & 14 & 43 & 527 \\
\hline \multicolumn{2}{|c|}{ Total Time } & 195.53 & 179.32 & 204.93 & 181.35 & 181.16 & 181.80 & 195.301 & 182.87 & 1502.26 \\
\hline
\end{tabular}




\section{References}

(1) Rockwell, T.J. (1988). Spare visual capacity in driving revisited: New empirical results for an old idea. In A.G. Gale (Ed.), Vision in vehicles II (pp 317-324). Amsterdam: North Holland.

(2) Wierwille, W.W., Antin, J.F., Dingus, T.A., \& Hulse, M.C. (1988). Visual attentional demand of an in-car navigation display system. In A.G. Gale (Ed.), Vision in vehicles II (pp 307-316). Amsterdam: North Holland. 


\section{Appendix 6A \\ Characterization of Driving Conditions \\ During the Data Collection Runs}

1. Posted Speed

$$
\mathrm{n}=31
$$

$45 \mathrm{mph}$

$55 \mathrm{mph}$

$65 \mathrm{mph}$

Total

$\begin{array}{rr}4 & 13 \% \\ 10 & 32 \% \\ 17 & 55 \% \\ 31 & 100 \%\end{array}$

2. Observed Speed

$$
\mathrm{n}=36
$$

$45 \mathrm{mph}$

$50 \mathrm{mph}$

$55 \mathrm{mph}$

$60 \mathrm{mph}$

60+ mph*

Total

$\begin{array}{rr}1 & 3 \% \\ 1 & 3 \% \\ 22 & 61 \% \\ 11 & 30 \% \\ 1 & 3 \% \\ 36 & 100 \%\end{array}$

*Note: not above $65 \mathrm{mph}$

3. $\quad \mathbf{n}=\mathbf{7 5}$

Day

Dusk

Night

4. Weather

Rain
Dry
$\quad$ Total

5. In a Construction Zone

$\begin{array}{rr}6 / 78 & 8 \% \\ 72 / 78 & 92 \% \\ & 100 \%\end{array}$

6. Traffic Density*

$$
\mathrm{n}=77
$$

$$
16 / 77=21 \%
$$

Light

Medium to Light

Medium

Heavy to Medium

Heavy

$$
\text { Total }
$$

$46 \quad 61 \%$

$6 \quad 8 \%$

$23 \quad 31 \%$

$$
\mathrm{n}=78
$$

$$
\mathrm{n}=77
$$

$\begin{array}{rr}32 & 42 \% \\ 6 & 8 \% \\ 25 & 32 \% \\ 2 & 3 \% \\ 12 & 16 \% \\ 77 & 101 \%\end{array}$

* Subjective estimate based on the number of vehicles in the road scene in direction of travel 
7. Most of the Observations were Taken on Rural Freeways (95\%)

8. Number of Lanes (going one-way) $\quad \mathrm{n}=77$

$\begin{array}{lrr}1 \text { Lane } & 3 & 4 \% \\ 2 \text { Lanes } & 58 & 75 \% \\ 3 \text { Lanes } & 16 & 21 \% \\ \text { Total } & 77 & 100 \%\end{array}$

9. Percentage of Observations Taken When Being

Passed by Another Vehicle $\quad \mathrm{n}=78$

Car $\quad 148 \%$

Truck $4 \quad 5 \%$

Not Passed $(100-23 \%)=77 \%$

10. Percentage of Observations Taken When Truck

Passed Another Vehicle $\quad \mathrm{n}=78$

Car $23 \%$

Truck $3 \quad 4 \%$

Not Passing $(100-7 \%)=93 \%$

11. Truck or Car Following* $\quad \mathrm{n}=78$

$\begin{array}{lcc}\text { Car } & & 228 \% \\ \text { Truck } & 7 & 9 \% \\ \quad \text { Total } & 29 & 37 \%\end{array}$

* Based on an estimate of vehicles ahead in the same lane. 
Appendix 6B

Truck Driver Workload Study

Subject Debriefing Summary

1. In comparing this run with others you have recently made, is there anything about the driving conditions today that wasn't typical? [Probe for unusual conditions about the road, traffic or weather that could effect workload.]

No problems

Excessive unloading at drop-off

8

1

2. How about the way the truck handled today?

Truck Handled Fine

9

3. For the rest of our discussion, I want you to think about workload in a particular way. When I use the word workload what I mean is how active you are when you're driving the truck and how much concentration you must devote to driving.

By active I mean how frequently you have to shift gears, steer, brake, change lanes, accelerate, check your mirrors, watch the traffic, listen for things, etc. Concentration simply means how much attention you must pay to driving to safely operate the truck.

Do you follow my definition? Workload is how active you are and how much you have to concentrate to drive the truck. Okay?

3a. Keeping in mind my definition of workload, can you think of any driving situations that involve high workload for you?*

$\begin{array}{ll}\text { None } & 3 \\ \text { Mountain driving } & 1 \\ \text { Bad weather } & 1 \\ \text { Heavy load } & 2 \\ \text { Black ice } & 1 \\ \text { Construction zones } & 1 \\ \text { Heavy traffic } & 2 \\ \text { Heavy traffic at exit ramps } & 1\end{array}$

3b. What exactly is it about the situation you reported that makes it high workload? Probe for driver, environmental, roadway, traffic and truck factors.

Lack of control over truck (going around corners and down hills)

Top heavy

Can't tell you are on black ice

Switching lanes (heavy traffic)

No comment

3
1
1
2
1
1
2
1

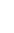
1

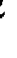


- $\mathrm{N}>9$ because of multiple responses

3c. On a scale of 1 to 10 , where 10 represents the most extreme case of high workload, how would you rate this situation?

3d. Do you do anything special to compensate for the higher workload when driving in this situation?

Heavy Load - 6

more careful, lack of control (going around corners and up/down hills), pay more attention, check equipment

Heavy Traffic - 6

switching lanes, pay more attention

Heavy Load - 8

top heavy going up/down hills, be more careful/slow down

Mountain Driving - 8

control the truck, stay alert/don't hurry

Black Ice - 10

hard to tell if you are on it; the road just looks wet, slow down/stop if needed

Construction Zones/Heavy Traffic - 10

pull over and wait

4. What one driving situation do you feel involves the most workload (i.e., the most driving activity and concentration) for all truck drivers, in general?*

Driving at night

Heavy traffic

Winter driving

Being considerate to other drivers

Knowing the limits of your driving

skills and the truck's capabilities

Too many hours in the day

Heavyrain/fog

Bad weather

5. Do you have any comments about our study?

No comment

I was not distracted

I enjoyed it

It was different

Hope your study makes a difference

There are things that can be done to improve 
trucks, e.g., external video cameras to reduce blind spots at front of truck

Great! Thanks for your help.

$* \mathrm{~N}>9$ because of multiple responses 


\subsection{PRELIMINARY ANALYSIS OF FEATURES OF IN-CAB DEVICES}

\section{Introduction}

To aid workload protocol development, a preliminary analysis of features of selected categories of in-cab devices was carried out. The intent of this work was to provide general guidance on the types of instrument features and phenomena might need to be factored into the protocol development. In addition, this type of preliminary analysis might help the project team decide what devices might be recommended to exercise the protocol and provide a broad range of assessment challenges.

\section{Objective}

The objective of this effort was to characterize, in a general way, key human factors attributes of selected categories of high technology in-cab devices. The analysis included features of devices which are believed to influence their use and associated workload. This preliminary analysis also provides some indication for the two types of devices that might be recommended for evaluation later in the project. Finally, the preliminary analysis provides some additional guidance for phenomena which the workload assessment protocol might profitably address.

\section{Procedure}

Using Burger, Smith, and Ziedman (1989) report as a starting point, Battelle staff contacted various incab device vendors for brochures and information on their products. Additional products were identified through an informal review of trade journals in the trucking industry and through professional contacts made by the Battelle team driver consultant. This provided the corpus of material which we reviewed for this assessment.

Based on the material collected, the following categories of devices were developed as representative:

- Voice communication systems

- Vehicle navigation and route guidance systems

- Single/integrated displays

- Text communication systems

Note that warning systems per se are absent from this list. While IVHS style warning systems are coming onto the market place (e.g., VORAD headway detection systems), they are currently not commonly available and so were excluded from consideration.

The approach for the preliminary task analysis was developed and reviewed with the NHTSA COTR. Table 7-1 indicates the types of questions which can have an impact on protocol development. These questions were used to guide the reviewers' assessment of devices within each of the above categories. 


\section{Protocol for NHTSA HV Analysis of In-Cab Device Features}

In-cab Device

Dimension

When used:

Relevant forward motion driving

tasks:

Driver-initiated or Device-initiated interaction:

Loads placed on driver:

Type of cognitive tasks required

Time required

Error modes

Task steps

Perseverance effects

\section{$\underline{\text { Relevance to Protocol Development }}$}

Indicates if device is used pre-trip, en route, only while parked, etc.

See Task 2 Interim Report for standard driving tasks. Will help identify reasonable times to present in-cab tasks.

Important to establish locus of control. Driver may better manage workload if device has driverinitiated interactions,

Visual, manual, cognitive loads are essential measures of the load imposed by an in-cab device.

E.g., check reading, text display, typing input, etc. would be useful information for assessing possibility of interference with primary driving task.

Important to note if an interaction is measured in fractions of a second, seconds, minutes, or portions of a hour. This will provide guidance on the necessary and sufficient timing properties of candidate workload measures.

What can go wrong and likely driver reactions. May provide indication of driver involvement with in-cab device transactions.

Indicated, perhaps, by mode of operation. May eventually be useful for explaining the locus of a workload effect.

Device allows driver to readily break with task and come back to it vs. design which prompts driver to persevere (e.g., because needed data is rolling off the screen, because system timeouts reset a control, etc.). This feature of devices is thought to have high relevance for highway safety. 
Importance of interaction:

Positioning:

Likelihood of use with other systems:
Perceived criticality (e.g., must do, optional); an indication of the urgency with which the driver will want to respond to the device.

Where device is mounted: on dash, overhead, on seat, other. May affect driver posture and lead to loss of visual awareness of the driving situation.

What other systems might be used with the device in question. E.g., a multifunction/integrated digital communications system may not be used with a trip recorder (because it already has that function built into it) but could be used with, say, a cellular phone. 


\section{$\underline{\text { Results and Conclusions }}$}

Results of the preliminary analysis are given in Tables 7-2 through 7-5. In general, this assessment suggests the importance of selecting devices for protocol testing which span a wide range of workload effects and on-the-road uses. In particular, it appears that a vehicle navigation system, combined with a voice-plus-text communication system would be a particularly good pair of devices to evaluate, both singly and in combination, They provide a wide range of workload measurement opportunities which will be incorporated into the to-be-developed protocol.

Future task analysis of specific products should pay close attention to lockouts in the design. Some manufacturers design their systems so that interaction is not possible while the truck is in motion. Other (e.g., TravTek) reduce the number or complexity of available interactions while the vehicle is in motion. Clearly, one aspect of the workload assessment protocol should be to support the judicious application of the lockout concept and identify conditions under which lockouts are most appropriate. 
Table 7-2

\section{Voice Communications Systems}

Examples: CB Radio, Cellular Telephone, "hands off" telephone, voice-only communications systems (e.g., Motorola System 36).

When Used:

Relevant forward motion driving tasks:

Driver-initiated or device initiated:

Loads placed on driver:

Types of Cognitive

Tasks:

Time required:

Error modes:

Task steps:

Perseverance
Virtually any time en route. Vehicle likely to be in motion.

While not intrinsic to any particular driving task or maneuver, communications may take place over a time interval in which many different driving tasks must be accomplished under varied driving conditions.

Can be either. Driver may call someone or be hailed by someone.

Device dependent. Could place significant amounts of the following:

- manual loads (for dialing, holding a handpiece, adjusting volume, etc.),

- $\quad$ visual loads (for manual dialing),

- visual-manual activities such as picking up the mic or receiver),

- cognitive loads (for engaging in conversation, comprehending instructions; nature of messages may be critical, e.g., spatial information vs. non-spatial information),

- auditory loads (for listening),

- vocal load (for vocally responding).
Varies for subtasks. Most time-consuming portion of the interaction is the conversation, measured in minutes or portions of an hour.

Wrong channel or number (to dial), misunderstanding what caller is saying, driver mispronouncing what is meant, volume inappropriate, squelch inappropriate, etc.)

Various, dependent on specific product. 
Effects :

Importance of Interaction:

Positioning:

Likelihood of use with other systems:

Overall Assessment:

Preliminary

Recommendations:
Could be substantial. Social/Organizational motivations to place a call, answer a call, keep with the conversation, etc. may lead driver to devote non-negligible attention to communication task.

Unclear how urgently the driver will want to respond or engage in the communication. Probably similar to answering the telephone at work or at office (e.g., medium to high). Perhaps similar to placing a call (for logging in, returning a call, etc.).

Various locations on dash, overhead, in area between seats (or dog house in cab-over), other locations. Most likely that the driver will manipulate the communications equipment with right hand.

High, especially as time goes on.

Communications systems are a class of high technology devices which could impose significant workload on heavy vehicle drivers. Visual and manual loads may be negligible for "hands off' designs. However, evidence exists for significant cognitive load. Auditory and vocal loads undetermined at this time.

This class of in-cab devices indicates a need for attentional workload measure which is not necessarily associated with visual allocation. Auditory load assessment probably needed for comprehensive assessment and may be indicative of attentional load (e.g., increase in "say again" or missed information). Vocal load assessment probably should not be measured independently of attentional load but may instead be indicative of attentional load (e.g., decreased fluency, slowed vocalization, increase in "uh's" overbase line/average). 
Table 7-3

\section{Vehicle Navigation and Route Guidance Systems}

Examples: ETAK Navigator, Blaupunkt TravelPilot

When Used: Virtually any time en route.

Relevant

Driving Tasks:

Driver-initiated

or Device-initiated:
Since these systems assist the driver in wayfinding, it is possible that they will be used prior to if not concurrently with such driving tasks as

- exiting using an exit ramp,

- merging into a traffic flow,

- changing lanes, and

- traveling through intersections

- making right and left turns at intersections.

Varies with specific systems. Systems with auditory display may "sound" out directions which the driver might need to hear again if he was not attending to the message initially. This would invite driver interaction directly. Similarly, the driver might elect to check his location or poll the system for route information when convenient.

Loads placed on Driver:

Varies with specific system design. Could place significant amounts of the following:

- manual loads (for menu selection, zooming/panning, etc.),

- $\quad$ visual loads (for map reading (spatial), text reading (e.g., labels), other),

- visual-manual activities such as guiding the hand over to the controls as units vibrate, visually controlling zoom and pan, etc. ,

- cognitive loads (for traversing a hierarchy of functions via programmable function keys, for comprehending maps (e.g., orienting and translating display to highway, other (e.g., error messages)),

- Auditory Loads (for listening to route instructions).

Types of Cognitive

Tasks: TBD

Time required:

Varies for subtasks. Most time-consuming portions of the interaction are likely to be manipulating programmable function keys, comprehending map display, comprehending vocal instructions. 
Error modes:

Task steps:

Perseverance

Effects:

Importance of

Interaction:

Positioning:

Likelihood of use with other systems:

Overall Assessment:

Preliminary

Recommendations:
Mode errors with programmable function keys; not understanding map display; not understanding auditory instructions (north vs. south rather than left vs. right); other.

Various, dependent on specific product.

Unknown but could be substantial at times. For example, if a particular system has a "rolling map" display, perhaps a critical waypoint is scrolling off the screen. The driver may persevere in trying to read the display before the critical information is lost (e.g., because it is perceived as too much trouble to scroll and pan back and forth, because that is not possible, etc.). For auditory displays, it may be that the driver did not understand or does not recall all of a message and so must invoke a "say again" function.

To the extent that the navigation system provides useful or essential wayfinding information, this device interaction will likely be considered highly important to heavy vehicle operations.

Various locations on dash, overhead, or between seats, wherever visual display can face the driver. Most likely that the driver will manipulate the navigation equipment with right hand.

Note: Al Turanski notes that since many in-cab tasks must be done with the right hand, drivers develop skill in left-hand only steering, especially with power steering.

High that other systems will be available, Not clear if other systems will be used concurrently with navigation systems.

Vehicle navigation and route guidance systems are another class of high technology devices which could impose significant workload on heavy vehicle drivers. Visual and manual loads are likely to be medium to high for many designs. Significant cognitive load may result from presentation of too much information. Individual differences in route familiarity, spatial processing abilities, and other factors may contribute to significant workload for a particular case. Auditory load could be high.

This class of in-cab devices is a recommended candidate for device evaluation in this project. In particular, navigation and 
route guidance systems indicate a need for the workload assessment protocol to address spatial information processing as a distinct message type. It is known that individual differences exist in spatial cognitive abilities (cf., Fleishman and Quaintance, 1984; Carroll, 1990) and preferred presentation mode for wayfinding information presentation (Streeter, Vitello, and Wonsiewicz, 1985). Therefore, the consideration of individual differences in the workload protocol should be addressed in light of the fact that we seek general understanding of the safety implications of in-cab devices. Color blindness could negate the decluttering potential of coloring coding.

Such studies have been performed at VP1 \& it was found that use of a land navigation system when the driver was required to select his or her own route created a workload-intensive situation [Antin, Ding-us, Hulse, \& Wierwille, 1990]. 


\section{Table 7-4 \\ Single/Integrated Displays (Status Displays)}

Examples:

When used:

Relevant forward motion driving tasks:

Drive-initiated or Device-initiated:

Loads placed on driver:

Types of

Cognitive Tasks:

Time required:

Error modes:

Task steps:

Perseverance

Effects:
Speedometer, tachometer, oil light, tire pressure, fuel reading, etc.

Virtually any time en route. Vehicle likely to be in motion.

Since these systems provide information concerning driver performance (e.g., speedometer) or vehicle status (e.g., oil light) they may be used at any time during the driving task.

Usually will be driver-initiated, i.e., driver may glance at display at will. However, display may flash or sound alarm if threshold reached (e.g., speedometer reads over $65 \mathrm{mph}$ ).

Device dependent, however, most likely types of load are:

- visual loads (e.g., search and identify display, discriminate/read presented information),

- cognitive loads (e.g., integrate received information into current schema, decide on appropriate course of action),

- auditory loads (if system uses auditory hailing),

- manual loads (none, unless driver must navigate through a menu hierarchy.

TBD

Fractions of a second, however, may vary depending upon complexity of display (search time) and "legibility" (difficulty of discrimination).

Mis-reading information, distraction from road if display flashes or has bright moving parts.

Move eyes (and possibly turn head). May be an action required to turn off alerting signals (if present).

Minimal since information available continuously, driver familiar with location of display and range of display values, and information usually of a discrete, simple variety. 
Importance of

Interaction:

Positioning:

Likelihood of use with other systems:

Overall Assessment:

Preliminary Recommendations:
Generally, low to moderately important. Displays may be essential to the driving task (e.g., speedometer) or others may indicate possible vehicle failure (e.g., oil light).

Various locations, most likely on dash and/or within peripheral view of driver while head facing forward.

High since other systems may make use of this information (e.g., speedometer for inertial navigation systems) or driver may need to make use of when communicating with other in-cab devices (e.g., record fuel reading into electronic log book).

Single or integrated status displays are not likely to produce significant workload demands on heavy vehicle drivers unless device-initiated communication distracts driver from road, or poorly designed, complicated displays require increased search times or significant mental operations to distill relevant information. Auditory alerts may introduce significant momentary load.

Single or integrated status displays do not, as a class, warrant evaluation in this project. Several factors including the familiarity of these devices to drivers, the usually loud visual load required, the need to integrate this information into the driving task thus allowing "preparation to receive" before visual saccade even begins, and many other factors make this the least potentially troublesome category reviewed. However, extremely cluttered, flashing or auditory hailing attachments, or radical changes in information display format (e.g., speed is indicated by color or brightness intensity) may require evaluation on an individualized basis. 
Table 7-5

Text Communication Devices

Examples:

When Used:

Relevant

Driving Tasks:

Driver-initiated or device-initiated:

Loads placed on driver:

Types of

Cognitive Tasks:

Time required:

Error modes:

\section{Task steps:}

Perseverance

Effects:
Sony 2-Wayfarer, Motorola CoveragePLUS, Hughes SkyRider

Virtually any time en route, however, some companies install devices facing away from driver so that they have to pull to side of road to read and respond to messages.

While not intrinsic to any driving task, communications may take place over a time interval in which many different driving tasks must be accomplished under varied driving conditions.

Can be either.

Device dependent. Could place significant amounts of the following:

- manual loads (e.g., keyboard, function key, or touch-screen entry) $?$

- visual loads (e.g., reading, locating response buttons),

- cognitive loads (e.g., comprehending instructions, composing reply, change-in-route planning),

- and an insignificant auditory load may be expected if device hails driver using tone or buzz.

TBD

Varies by subtasks. Time lapses best measured in minutes may occur for composing reply, typing in reply, or interactive messaging with dispatcher.

Misreading display, mistyping reply, misidentifying auditory hailing cue.

Various dependent on specific product.

Could be substantial. If message scrolls driver must pay continuous attention to receive full message. Amount of text displayed may encourage increased dwell times to read entire message in one look. Urgent reply may require continuous attention to type in message. Drivers may be tempted to communicate with devices while driving even if instructed not to. 
Importance of Interaction:

Positioning:

Likelihood of use with other systems:

Overall

Assessment:

Preliminary

Recommendations:
Unclear how urgently the driver will want to respond or engage in the communication. Probably similar to answering messages at work. Organizational protocols may require driver-initiated communication at specific points or times en route.

May be positioned on dash, overhead, between seats, or facing away from driver in sleeper or on passenger side. If within reach of driver, most likely manipulated with right hand.

High. If positioned so that available to driver during driving, can expect it may be used at any time en route.

Text communication devices are a class of high technology devices which could impose significant workload on heavy vehicle drivers. Visual and manual loads for communicating with these devices are expected to be high. Amount of text displayed on screen may be a significant determiner of dwell time (perseverance effects). Mounting of device so that it is inaccessible during driving may significantly reduce workload.

This class of in-cab devices places significant load on visual and manual resources. Eye-glance techniques and hands-off-wheel techniques are most likely candidates for workload assessment. Because these devices are not integral to moment-by-moment driving requirements, they may be mounted inaccessible to driver. Therefore, summary workload demands will likely be contingent upon mounting arrangement. It is noteworthy that text messages may place a substantial level of visual or cognitive load on the driver. 


\section{References}

(1) Antin, J.A., Dingus, T.A., Hulse, M.C., \& Wierwille, W.W. (1990). An evaluation of the effectiveness and efficiency of an automobile moving-map navigational display. Internal Journal of Man-Machine Studies, j3, 581-594.

(2) Carroll, J.B. (1990). The ETS Factor-Referenced Kit in the light of new reanalyses of classic factor-analytic studies. Paper uresented at the Annual Meeting of the American Educational Research Association, Boston.

(3) Fleishman, E.A., \& Quaintance, M.K. (1984). Taxonomies of human performance. Orlando, FL: Academic Press, Inc.

(4) Streeter, L.A., Vitello, D., \& Wonsiewicz, S.A. (1985). How to tell people where to go: Comparing navigational aids. International Journal on Man-Machine Studies, 22, 549-562. 


\subsection{SUMMARY}

The objective of this data collection effort was to collect task analytic data to support heavy vehicle driver workload assessment protocol development. Data collected included subjective assessments from drivers, visual allocations while driving, and manual activities while driving. These data provide useful insights into the following issues:

- $\quad$ The meaning of the term "workload" to heavy vehicle drivers;

- $\quad$ The demand placed on the driver by various driving conditions;

- The safety criticality and difficulty of selected standard driving tasks;

- $\quad$ The perceptual, motor, and cognitive loads imposed by various tasks and in-cab behaviors while on the road under various driving conditions; and

The features of selected high-technology in-cab devices and the loads which may be placed on drivers.

Key findings and their implications are provided below.

When professional heavy vehicle drivers think of "workload", they rather consistently speak of stress caused by delays to their schedules. Heavy traffic, inclement weather, and construction zones were most often mentioned as relative to perceived high driving workload. Interestingly, drivers often cited 'pay more attention to driving' as a primary way to cope with this added stress.

Given the frequency with which drivers encounter delays, scenarios included in the to-be-developed workload assessment protocols should incorporate some means of inducing time stress to make baseline studies and evaluations more realistic. Furthermore, special conditions such as construction zones would also be valuable to include in protocol scenarios to introduce realistic levels of driving task workload.

Apart from the global impact of schedule delays and operating practices, primary driving task demand is determined, to a large extent, by driving conditions outside the cab of the vehicle. A psychological scaling approach was taken to determine the relative importance of five driving condition factors on the demand placed on the driver. This approach allowed a unidimensional scale to be constructed with various combinations of these factors positioned along it. In decreasing order of their impact on demand, results included: traction, visibility, traffic, roadway type, and lighting. Furthermore, traction loss and poor visibility were weighted consistently and substantially as more important to the level of driving condition demand a driver faces. From this, we conclude that, to create high demand in the driving task, traction, visibility, and traffic density should be manipulated. These factors should be factored into simulator study design when feasible. However, the importance of appropriately scheduled on-the-road data collection to capture the effects of these demands is underscored. Finally, in-cab devices which are likely to be used in inclement weather (e.g, In-Vehicle Safety Advisory and Warning Systems (IVSAWS) must be evaluated in appropriately stringent conditions.

Drivers rated the safety criticality and difficulty of various driving tasks (e.g., lane change, merge, make left turn, etc.) in each of three sets of driving conditions. These three driving conditions were drawn such that they represented what were thought to be very demanding, moderately demanding, and minimally demanding driving conditions. Results, indicated, however, that the moderately and very 
demanding driving conditions were closer to each other than apparent from the unidimensional demand scale. Consistent with the demand analysis, however, was the result that tasks which require high visual/perceptual performance and precise or quick steering/braking control were rated as most difficult and most important to safety. From this, we conclude that protocol scenarios should be developed with opportunities to observe performance during such driving tasks.

On-the-road data collection allowed us to develop feasible techniques for recording visual workload data. Data from nine drivers were collected and visual allocation was measured along with driver manual activity. These measures were demonstrated to be sensitive to individual differences among the driver sample observed and across observed driving conditions. Baseline data were collected for common incab tasks in vehicles not equipped with high technology devices. Such data can serve as benchmarks against which to compare loads associated with high technology devices.

A preliminary analysis of selected high technology in-cab devices was also conducted. Key features suggest the importance of including certain types of measures in the workload assessment protocol. For example, cognitive load should be measured independent of visual allocation in some instances, e.g., hands-free voice communication. Furthermore, a key attribute of devices is likely to be how their design invites the driver to persevere and complete the transaction. This preliminary assessment provides indication that for verification and validation of the to-be-developed protocol, a vehicle navigation and route guidance system together with a voice-plus-text communication system will provide a wide range of workload measurement challenges. 